\title{
Dependence of excitability indices on membrane channel dynamics, myelin impedance, electrode location and stimulus waveforms in myelinated and unmyelinated fibre models
}

\author{
Thomas Tarnaud · Wout Joseph · Luc Martens · Emmeric Tanghe
}

Received: date / Accepted: date

\begin{abstract}
Neuronal excitability is determined in a complex way by several interacting factors, such as membrane dynamics, fibre geometry, electrode configuration, myelin impedance, neuronal terminations,... This study aims to increase understanding in excitability, by investigating the impact of these factors on different models of myelinated and unmyelinated fibres (five well known membrane models are combined with three electrostimulation models, that take into account the spatial structure of the neuron). Several excitability indices (rheobase, polarity ratio, bi/monophasic ratio, time constants,...) are calculated during extensive parametersweeps, allowing us to obtain novel findings on how these factors interact, e.g. how the dependency of excitability indices on the fibre diameter and myelin impedance is influenced by the electrode location and membrane dynamics.

It was found that excitability is profoundly impacted by the used membrane model and the location of the neuronal terminations. The approximation of infinite myelin impedance was investigated by two implementations of the spatially extended nonlinear node model. The impact of this approximation on the time constant of strength-duration plots is significant, and most important in the Frankenhaeuser-Huxley membrane model for large electrode-neuron separations. Finally, a multicompartmental model for C-fibres is used to determine the impact of the absence of internodes on excitability.
\end{abstract}

T. Tarnaud

Tel.: +32-471-85-30-53

E-mail: thomas.tarnaud@ugent.be

T. Tarnaud · W. Joseph $\cdot$ L. Martens $\cdot$ E. Tanghe

Ghent University-IMEC, Technologiepark 15, 9052 Zwijnaarde, Belgium
Keywords Electrostimulation · A-fibres · C-fibres · strength-duration $\cdot$ rheobase

\section{Introduction}

Two different approaches have been investigated in literature to model neuronal activity: mathematical IF ("integrate-and-fire") models and detailed biophysical models of the Hodgkin-Huxley (HH) type [12]. The former type of model has a low computational cost [16], allowing for large scale simulations, such as performed by Izhikevich and Edelman [17]. It was furthermore noted in literature that the IF-model is more realistic than a single-compartment Hodgkin-Huxley model, in which channel properties are constrained by patch-clamp measurements [3]. However, a multi-compartmental model of the HH-type has more empirical content (e.g. description of ionic currents) and is potentially more realistic than IF-models. Furthermore, computational models of the Hodgkin-Huxley type have biophysical meaningful and measurable parameters. E.g., in the blue brain project HH-type models are used to reproduce in vivo and in vitro experiments without parameter tuning [19].

In this paper, multi-compartmental HH-type models are used to investigate the dependence of excitability indices on the used equation set for the membrane dynamics and on the electrode location. Each membrane model is combined with one of the multi-compartmental models listed in Table 1, yielding a total of 15 different numerical electrostimulation models. An overview of the five used membrane models is shown in Table 2. The Hodgkin-Huxley (HH) model was the first mathematical (physiological) model to describe the excitable membrane and was obtained from voltage-clamp experiments on the squid giant axon [13]. Since then, var- 


\begin{tabular}{llc}
\hline Model & Description & Applicability \\
\hline SENN-MA & Crank-Nicolson implementation of spatially-extended non-linear node & Myelinated axons \\
& model [30]. All nodes non-linear. Myelin capacitance and conductance & \\
& neglected. & Myelinated axons \\
SENN-M & Compartmental representation of myelin included & Unmyelinated axons
\end{tabular}

Table 1 Spatially extended electrostimulation models

ious researchers have derived similar electrodynamical models from different types of excitable tissue. Frankenhaeuser and Huxley [10] (FH-model), Chiu et al. [7], and Sweeney et al. [38] (CRRSS-model), Schwarz and Eikhof [35] (SE-model), and Schwarz et al. [36] (SRBmodel) derived their models from myelinated frog, rabbit, rat, and human axons respectively. These membrane models (summarized in Table 2) are selected, because they are the "classical" models of membrane dynamics, that are often used for applications in which no specialized model exists or is accepted. Because of their widespread use in computational neuroscience (e.g., in computational studies of the cochlear nerve $[5,6,23]$ or for modeling conduction block [39, 42, 43]), these membrane models are especially interesting for this study, which aims to determine the influence of membrane dynamics on neuronal excitability.

The first multi-compartmental model (SENN-MA) listed in Table 1, describes a myelinated axon and is an implementation of the spatially extended nonlinear node (SENN) model [30]. It extends the original electrostimulation model proposed by McNeal, by including non-linear FH-equations for all nodes of Ranvier [22]. The SENN-model has been widely used by researchers to study electrostimulation of myelinated fibres and is used by IEEE C95.6 to derive basic restrictions for electromagnetic exposure [15]. As a consequence, ICNIRP (International Commission on Non-Ionizing Radiation Protection) has implicitly adopted results of the SENN model as well, by using basic restriction parameters from the IEEE standard [14, 31].

In the context of exposure assessment and electromagnetic safety, a comparison between excitability indices obtained by different fibre and membrane models is especially relevant. A recent publication on the research agenda of the IEEE International Committee on Electromagnetic Safety has listed 25 issues when using numerical models to derive electromagnetic basic restrictions, one of these on the consistency of excitation models [31]. Furthermore, a survey has been published, comparing the excitation thresholds obtained by neurostimulation models of different researchers [28]. As it was observed that excitation threshold can vary significantly between different electrostimulation models, a thorough investigation of the effect of the membrane dynamics, fibre diameter, myelin impedance, electrode location and stimulus waveform on excitability indices (e.g., strength-duration and rheobase) is important.

It was noted by McNeal that the approximation of negligible conductivity and capacity values of the myelin layer is the cause of the most serious error in his model [22]. This assumption, that we will call the myelin approximation (MA), is implicit to the SENNmodel as proposed by Reilly et al. [30]. One of the goals of this paper is to assess the impact of the myelin approximation on excitability indices. To this end, a second model (SENN-M) is used that incorporates additional sets of differential equations to account for the finite myelin parameters, that are distributed along the internodes. In this model, the capacity and conductivity values of myelin reported by Tasaki are used (Appendix A) $[40]$.

We note that also the SENN-MA model departs from the original SENN-model in this study, after substituting the Frankenhaeuser-Huxley equation set with one of the membrane dynamical models from Table 2. This procedure was for instance used by Cartee to compare the effect between different membrane models on the summation and refraction properties of the modeled neuron [5].

Finally, a model for unmyelinated fibres (termed "CFIBRE" in Table 1) is obtained by carefully altering the geometrical and electrophysiological parameters of the SENN-M model. Models of unmyelinated fibres, similar to the C-FIBRE model used in this study, have been used by researchers to study the excitation properties of C-fibres [39, 43, 11]. Model equations for the different fibre and membrane models (Table 1-2) used in this study are summarized in Appendix A.

\begin{tabular}{lll}
\hline Model & Abbr. & Experiments \\
\hline Hodgkin-Huxley & HH & Squid axon \\
Frankenhaeuser-Huxley & FH & Frog node \\
Chiu-Ritchie-Rogart-Stagg- & CRRSS & Rabbit node \\
Sweeney & & \\
Schwarz-Eikhof & SE & Rat node \\
Schwarz-Reid-Bostock & SRB & Human nerve
\end{tabular}

Table 2 Models of membrane channel dynamics and their corresponding abbreviations (abbr.) 
The first goal of this paper is to investigate the dependence of excitability indices on the used equation set for the neuronal membrane, the electrode location, and stimulus properties. Simulation results of the strength-duration relation, rheobase, polarity ratio and bi/monophasic ratio obtained by the different models (summarized in Table 1 and Table 2) are presented and compared in the following sections. Correspondence of strength-duration (SD) plots, obtained by different membrane models and electrode locations, with WeissLapicque and Lapicque-Blair fits is subsequently investigated. Furthermore, the impact of the presence of neuronal terminations on excitability is considered. The second goal is to determine the impact of the myelin approximation in the SENN-model on the excitability indices, for different electrode locations and models of membrane dynamics. The third goal is to compare excitability indices among different fibre models (SENNMA, SENN-M, and C-FIBRE). The impact of the absence of internodes in the C-FIBRE model on neuronal excitability is determined. To this end, all geometrical and physiological parameters (i.e. all parameters, except for the spatial and temporal discretisation step and simulation time) are first held constant, to allow comparison between the SENN-M and C-FIBRE model for the FH, CRRSS, SE, and SRB membrane models (see also next section). In a second step, the impact of the fibre diameter on the rheobase and strength-duration time constant $\tau_{e}$ in C-fibres is determined.

\section{Methods}

Computational models for a myelinated axon (SENNMA, SENN-M) and an unmyelinated axon (C-FIBRE) are implemented in Matlab. In each of these models, the corresponding system of equations is discretized by a Crank-Nicholson scheme (see Appendix A) [8], while the ends of the neuron are assumed to be sealed (i.e. no axonal current at the neuronal terminations). An overview of the used parameter values is shown in Table 3. All other parameters, not listed in Table 3, are obtained from literature and summarized in Appendix A. Experimental values for the myelin capacitance and
$(0,0)$

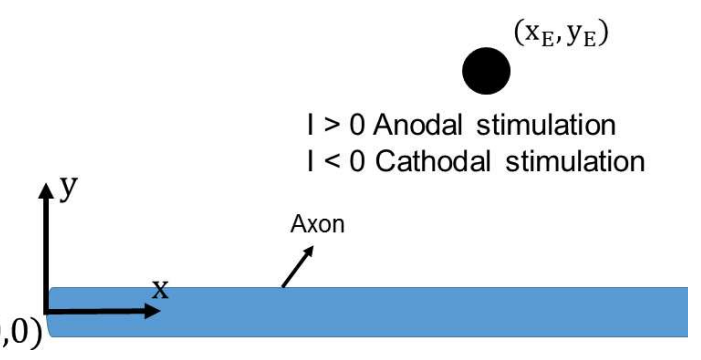

Fig. 1 Schematic representation of the simulation configuration. A current $I$ is injected by a spherical electrode into the extracellular fluid. The electrode is placed at longitudinal distance $x_{E}$ and radial distance $y_{E}$

conductance are obtained from Tasaki [40]. Other physiological and geometrical parameters are taken from the original McNeal publication and the appropriate papers describing the active membrane models $[22,13,10,7$, $38,35,36]$.

For all models (A-fibres and C-fibres) a $10 \mu \mathrm{m}$ neuron of length $L=4 \mathrm{~cm}$ is used, except when the SD time constant $\tau_{e}$ or rheobase $I_{b}$ are explicitly plotted as function of the diameter $D$. As the propagation speed of action potentials is lower in C-fibres than in A-fibres, while activation thresholds are higher, a larger (maximal) temporal discretization step of $10 \mu$ s could be used in the C-FIBRE model. For A-fibres, a maximal temporal discretisation step of $1.26 \mu$ s was used. Furthermore, the temporal discretization step is altered for low pulse durations $t_{p}$, in order to ensure a minimum of 10,50 , and 70 samples per pulse in the C-FIBRE, SENN-MA, and SENN-M model respectively. The total simulationtime $T_{s i m}$ is at least six times the phase duration (i.e. $T_{\text {sim }} \geq 6 \tau_{p}$, where $\tau_{p}$ is the duration of a single phase). Finally, the simulation time is not allowed to be lower than $1 \mathrm{~ms}$ for A-fibres and $5 \mathrm{~ms}$ for C-fibres. These requirements are necessary to ensure sufficient simulation time to allow the propagation of the action potential and were determined by simulation of generic electrode configurations. Higher simulation times are required for

\begin{tabular}{llll}
\hline Variable & Description & A-fibre & C-fibre \\
\hline$\Delta x$ & Spatial discretisation step & $0.1 \mathrm{~mm}$ & $0.1 \mathrm{~mm}$ \\
$\Delta t$ & Temporal step & $1.26 \mu \mathrm{s}$ & $0.01 \mathrm{~ms}$ \\
$\mathrm{D}$ & Outer radius & $10 \mu \mathrm{m}$ & $10 \mu \mathrm{m}$ \\
$\mathrm{d}$ & Inner radius & $0.7 \mathrm{D}$ & $\mathrm{D}$ \\
$\mathrm{L}$ & Neuron length & $4 \mathrm{~cm}$ & $4 \mathrm{~cm}$ \\
$T_{s i m}$ & Simulation time & $\geq \max \left(6 \tau_{p}, 1 \mathrm{~ms}\right)$ & $\geq \max \left(6 \tau_{p}, 5 \mathrm{~ms}\right)$ \\
$\mathrm{T}$ & Temperature & $18.5^{\circ} \mathrm{C}$ & $18.5^{\circ} \mathrm{C}$
\end{tabular}

Table 3 Values and description of parameters of the A-fibre and C-fibre model 


\begin{tabular}{llll}
\hline Type & Pulse-duration $\tau_{p}$ & Rad. distance $y_{E}$ & Long. distance $x_{E}$ \\
\hline SD-plot $I_{0}\left(\tau_{p}\right)$ & $5 \mu \mathrm{s}-5 \mathrm{~ms}(31, \log )$. & $2 \mathrm{~mm}-1 \mathrm{~cm}$ & $2 \mathrm{~cm}$ \\
& $5 \mu \mathrm{s}-5 \mathrm{~ms}(31, \log )$. & $5 \mathrm{~mm}$ & $0.5 \mathrm{~cm}-2 \mathrm{~cm}(4, \operatorname{lin})$. \\
$R_{b m}$ & $5 \mu \mathrm{s}-5 \mathrm{~ms}(31, \log )$. & $2 \mathrm{~mm}-1 \mathrm{~cm}$ & $2 \mathrm{~cm}$ \\
Rheobase $I_{b}$ & $5 \mathrm{~ms}$ & $1.5 \mathrm{~mm}-10 \mathrm{~mm}(10$, lin. $)$ & $0.5 \mathrm{~cm}-2 \mathrm{~cm}(4, \operatorname{lin})$. \\
Polarity ratio $R_{p}$ & $5 \mathrm{~ms}$ & $2 \mathrm{~mm}-1 \mathrm{~cm}$ & $0.5 \mathrm{~cm}-2 \mathrm{~cm}(10$, lin.)
\end{tabular}

Table 4 Simulation range of electrode and waveform parameters used in different types of plots. The number of simulation points and the used distribution for these points (linear (lin.) or logarithmic (log.)) is indicated between brackets. $I_{0}$ is the threshold current, $R_{b m}$ is the bi/monophasic ratio, $I_{b}$ is the rheobase, and $R_{p}$ is the polarity ratio

C-fibres due to low propagation speeds of the action potential.

As the Hodgkin-Huxley model was obtained from an unmyelinated squid nerve, axonal currents are too weak to sustain the propagation of an action potential in the framework of the SENN-model $[5,9]$. This phenomenon is caused by the higher axonal resistance between neighbouring compartments in the SENN-model, as compared with the C-FIBRE model. However, combination of the Hodgkin-Huxley with a myelinated fibre model has proven useful, e.g. in modeling the auditory nerve [23]. Consequentially, the Hodgkin-Huxley equations were modified to allow compatibility with the SENN-model. The modifications were performed, following the procedure used by Cartee [5], which is based on Fitzhugh [9]. To allow for conduction of an action potential when the Hodgkin-Huxley model is combined with the SENN-model, a scaling factor $A_{s c}=$ $0.003 \mathrm{~mm}^{2}$ is used. The nodal conductivity and capacitance for the SENN-model with HH membrane dynamics is scaled with $A_{s c} /\left(\pi d l_{n}\right)$ and $A_{s c} /\left(20 \pi d l_{n}\right)$ respectively. Here, $l_{n}=2.5 \mu \mathrm{m}$ is the nodal length. It is noted that $A_{s c}$ has no physical significance and should be interpreted as a scaling factor to match model parameters with the experimentally measured conductivity and capacitance. This modification of the Hodgkin-Huxley equations, when applied to the SENN-model, was the only deviation of the original membrane parameters and is only applied when the HH-equations are used in combination with the SENN-model.

A different phenomenon observed by Rattay and Aberham is known as "heat block". Unmodified HHequations stop to conduct action potentials for temperatures greater than $31^{\circ} \mathrm{C}$ [27]. The modified HHequations that are used in the SENN-model will not be hindered by heat block [5]. However, unmodified Hodgkin-Huxley equations are still used in this study, in the C-FIBRE model. Because it is desired to compare all models at the same temperature, a universal temperature of $18.5^{\circ} \mathrm{C}$ is used for all models.

A spherical micro-electrode is used to probe the excitation properties of the model under consideration. A schematic representation of the simulation configuration, defining the longitudinal and radial electrode distance $x_{E}$ and $y_{E}$, is shown in Fig. 1. The minimal stimulation current $I$ required to obtain activation is determined by a titration procedure alternating between activation and absence of activation [29]. The titration procedure terminates after reaching an accuracy of $1 \%$ or better. Neuronal activation was defined as depolarization of the active membrane at subsequent active compartments. Electrode parameters that are varied are the electrode location, the pulse duration $\tau_{p}$ and the sign of the current (anodic or cathodic). Furthermore, both monophasic and biphasic square waves are used as stimulation pulses.

The excitability indices, used in this study, are now defined. Strength-duration plots represent the relation between the pulse duration $\tau_{p}$ and the threshold current $I_{0}$ required for neuronal activation. An empirical strength-duration relationship was first obtained by Weiss [41] and by Lapicque [18]. The Weiss-Lapicque relation between the stimulus strength and the pulse duration can be formulated as:

$I_{0}=I_{b}\left(1+\frac{\tau_{e}}{\tau_{p}}\right)$

Here, $I_{b}$ is the rheobase current and $\tau_{e}$ is the SD time constant.

Another strength-duration relation is based on theoretical considerations, and was obtained by Lapicque and Blair [18, 1, 4]. The Lapicque-Blair relation has an exponential form:

$I_{0}=\frac{I_{b}}{1-\exp \left(-\frac{\tau_{p}}{\tau_{e}}\right)}$

These equations define the rheobase current as the threshold current for a square-wave pulse of long duration. The rheobase is obtained in this study by evaluating the SD-plots at $\tau_{p}=5 \mathrm{~ms}$, similarly as is done in Reilly [28]. This procedure results in a negligible error on the rheobase, which is defined as the asymptotic minimum of the SD-plot and will be slightly lower for longer pulse durations. 
Similarly, the strength-duration time constant $\tau_{e}$ was approximated by taking the ratio of the charge $Q_{e}=5 \mu s \cdot I_{0}\left(\tau_{p}=5 \mu s\right)$ with respect to the rheobase $I_{b}$.

The bi/monophasic ratio $R_{b m}$ is defined as the ratio of the biphasic and monophasic threshold current. To determine these thresholds, a cathodal monophasic square wave is used, while the biphasic square wave will start with a cathodal initial phase. Note that the time $\tau_{p}$ indicates the duration of the initial phase in a biphasic square wave and not the duration of the pulse.

Finally, the polarity ratio $R_{p}$ is the ratio of the anodic and cathodic rheobase. The polarity ratio was approximated by evaluating the anodic and cathodic threshold current at pulse durations of $\tau_{p}=5 \mathrm{~ms}$ [28]. Values of $R_{p}$ lower than one indicate that anodic stimulation is more effective than cathodic stimulation to initiate the propagation of an action potential, and vice versa for $R_{p}>1$.

The simulation range of the electrode and waveform parameters, that are used to generate the plots in this paper, is summarized in Table 4 . The table also indicates the number of samples in the simulation range and whether a linear (lin.) or logarithmic (log.) distribution of points is used.

\section{Results}

An example of an action potential in the SENN-model with FH-dynamics is shown in Fig. 2. The anode is placed at the centre of the axon, at $x_{E}=20 \mathrm{~mm}$. The activating function $f$ allows for a straightforward interpretation of the location of the excitation node, which is defined as the first node to reach the voltage threshold [25]. The activating function is shown in Fig. 2(e)-(f) and is given by:

$$
\begin{array}{r}
f(l)=\frac{1}{C_{m}^{*}(l)}\left[G_{a, f}^{*}(l-\Delta l)\left(V_{e}(l-\Delta l)-V_{e}(l)\right)+\right. \\
\left.G_{a, b}^{*}(l+\Delta l)\left(V_{e}(l+\Delta l)-V_{e}(l)\right)\right]
\end{array}
$$

Here $C_{m}(l)^{*}$ is the membrane capacitance of the compartment at $x=l . G_{a, b}^{*}(l)$ is the "backward" axial conductance between the compartments at $x=l-\Delta l$ and $x=l$. Similarly, $G_{a, f}^{*}(l)$ is the "forward" axial conductance between the compartments at $x=l$ and $x=l+\Delta l$. Equation (3) is valid for all nodes, except the termination nodes at $x=0 \mathrm{~mm}$ and $x=40 \mathrm{~mm}$. At the neuronal terminals, the activation function $f$ is given by [26]:

$$
f(0)=\frac{2}{C_{m}^{*}(0)}\left[G_{a, b}^{*}(\Delta l)\left(V_{e}(\Delta l)-V_{e}(0)\right)\right]
$$
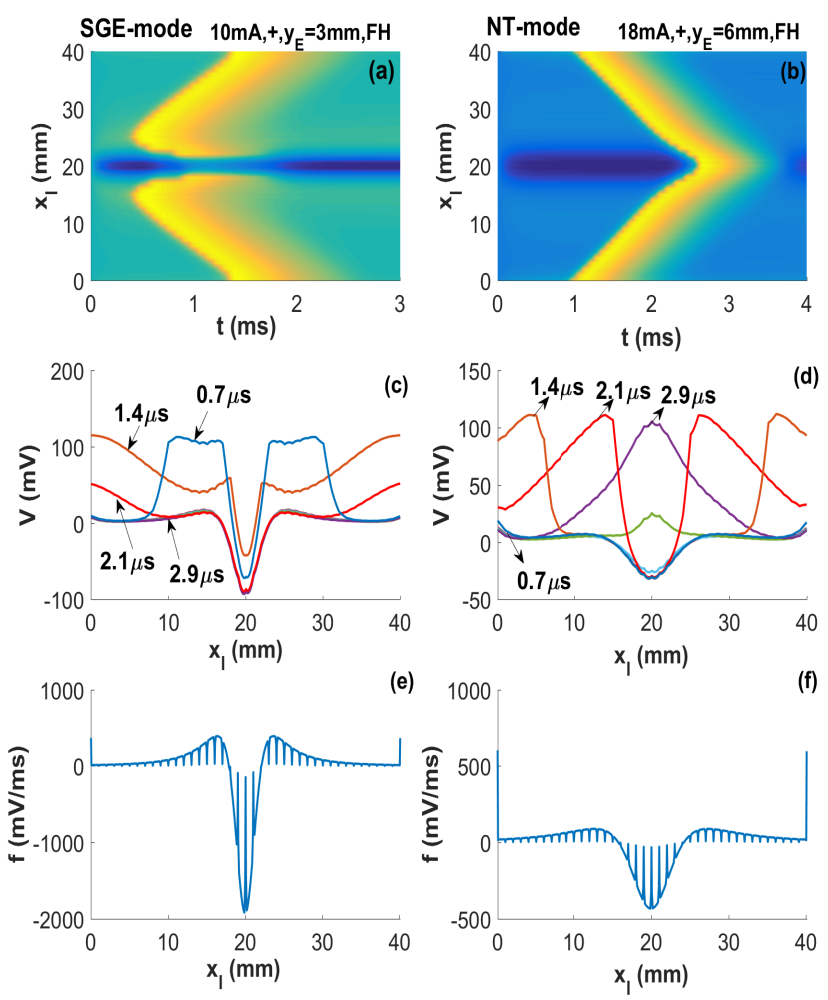

Fig. 2 Illustration of propagation of AP for the SGEmode (a,c,e) and NT-mode (b,d,f) of activation (see text for definitions). The anode is placed at longitudinal distance $x_{E}=20 \mathrm{~mm}$. (a)-(b) Colour map of membrane potential. Yellow indicates depolarization, dark blue indicates hyperpolarization. The abscissa represents the time $t$ passed after the onset of the stimulus, while the ordinate $x_{l}$ represents the length along the neuron. (c)(d) Spatial distribution of membrane voltage along the neuron for 7 snapshots, equally spaced in time (snapshots at the four earliest times are indicated). (e)-(f) Spatial distribution of activating function $f$

$$
f(L)=\frac{2}{C_{m}^{*}(L)}\left[G_{a, f}^{*}(L-\Delta l)\left(V_{e}(L-\Delta l)-V_{e}(L)\right)\right]
$$

Here, the factor 2 is a consequence of the fact that sealed-end boundary conditions are applied at the centre of the compartments at the neuronal terminations.

Negative values of the activating function will favor hyperpolarization of the active membrane, while the membrane will tend to be depolarized at compartments with positive values of $f$. It can be observed from Fig. 2(e)-(f), that the envelope of the activating function reaches a central minimum at $x_{E}=20 \mathrm{~mm}$ and two maxima at the so-called "virtual cathodes". These virtual cathodes are positioned symmetrically, at both sides of the anode. Furthermore, positive peaks at both neuronal terminations can be observed. 

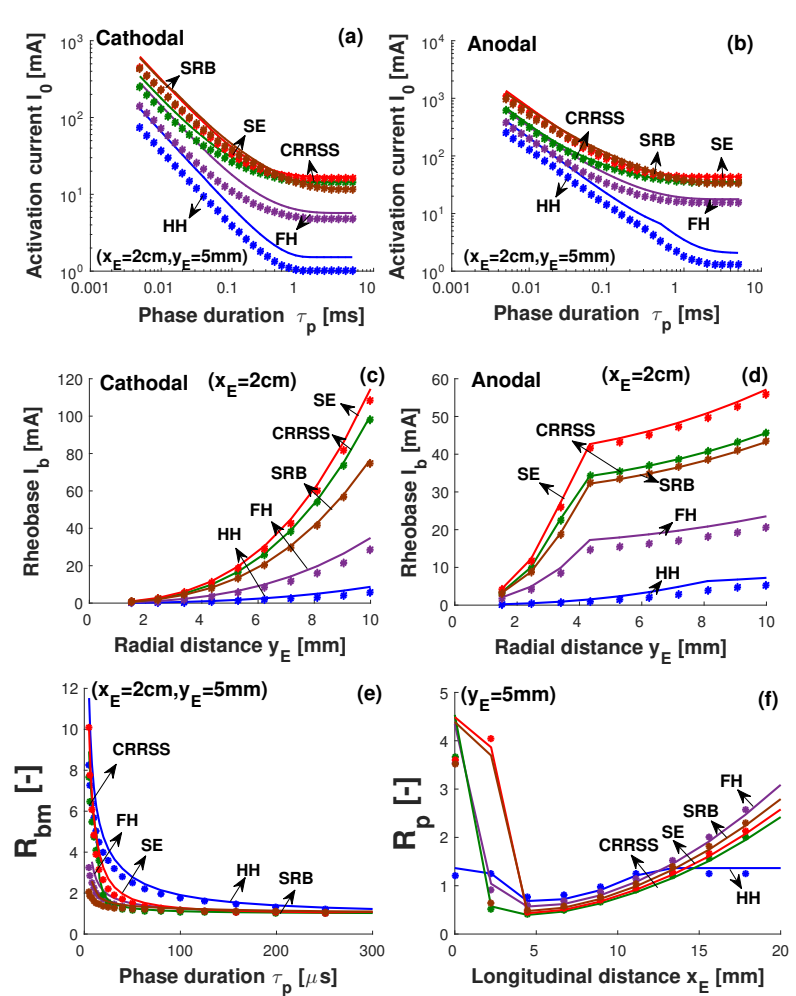

Fig. 3 Myelinated (A-fibre) simulation results. (a) cathodal activation current, (b) anodal activation current, (c) cathodal rheobase, (d) anodal rheobase, (e) bi/monophasic rheobase ratio, (f) polarity ratio. Full lines indicate the use of the myelin parameters reported by Tasaki [40], while the stars indicate the use of the myelin approximation

When the anode is placed closed to the axon, e.g. at $y_{E}=3 \mathrm{~mm}$ in Fig. 2(a),(c),(e), neuronal activation will be determined by the virtual cathodes. Because the activating function $f$ is proportional to the spatial gradient of the electric field (SGE) in equation (3), we will call this case the SGE-mode of activation. The excitation nodes are roughly located at the virtual cathodes and the action potential propagates orthodromically and antidromically. Propagation to the axon centre $\left(x_{E}=20 \mathrm{~mm}\right)$ is inhibited by the hyperpolarized region at the position of the anode.

Conversely, if the anode is placed at larger radial distance, e.g. at $y_{E}=6 \mathrm{~mm}$ in Fig. 2(b),(d),(f), then excitation will be driven by the large peaks of the activating function $f$ at the neuronal terminations. The action potential in Fig. 2(b),(d) starts at the neuronal terminations and propagates towards the centre $\left(x_{E}=20 \mathrm{~mm}\right)$. We will refer to this mode of activation as the neuronal termination (NT) mode.

For cathodal stimulation the sign of the activating function $f$ is reversed and the excitation node coincides with the location of the cathode.

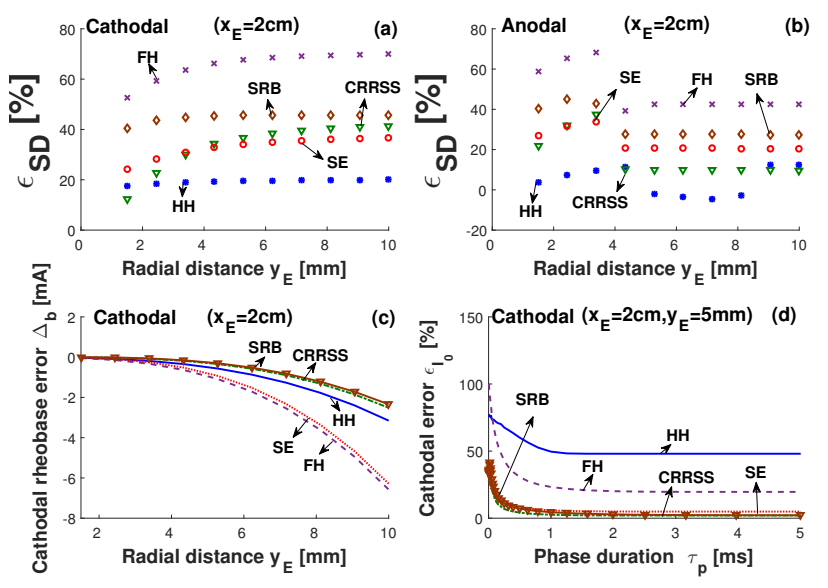

Fig. 4 Effect of the myelin approximation on excitability indices. (a)-(b) Relative error on $\tau_{e}$. (c) Relative error on cathodal threshold current. (d) Absolute error on cathodal rheobase. Electrode location is $x_{E}=2 \mathrm{~cm}$ in (a)-(d), and $y_{E}=5 \mathrm{~mm}$ in (d).

\subsection{A-fibres}

The effect of the myelin approximation and the membrane channel dynamics on excitability indices in the SENN-MA and SENN-M model is shown in Fig. 3. Fig. 3(a) and Fig. 3(b) are the cathodal and anodal SDplots obtained by running a parameter sweep over 31 logarithmically distributed pulse-durations in the interval from $5 \mu$ s to $5 \mathrm{~ms}$. Fig. 3(c)-(d) depict cathodal and anodal rheobase-distance plots respectively, obtained by simulation of 10 linearly distributed radial (rad.) distances, while the longitudinal (long.) distance is kept constant. Fig. 3(e)-(f) show the bi/monophasic ratio $R_{b m}$ and the polarity ratio in 31 logarithmically and 10 linearly distributed points respectively.

\section{Impact of the myelin approximation on excitability in- dices}

The influence of the myelin approximation on excitability indices was determined by Richardson et al. [32], for neurons described by a modification of the SchwarzReid-Bostock model [20]. We expand some of their results in this subsection, to include the electrode range, excitability indices and membrane models that are considered in this study.

Fig. 3 and Fig. 4 are used to illustrate the impact of the myelin approximation. Strength-duration curves shift to lower threshold current values, when the myelin conductance and capacitance are set to zero. This effect is most significant for shorter time durations (Fig. 3(a)(b), Fig. 4(d)). The observation that shorter time durations are associated with greater errors, is a conse- 

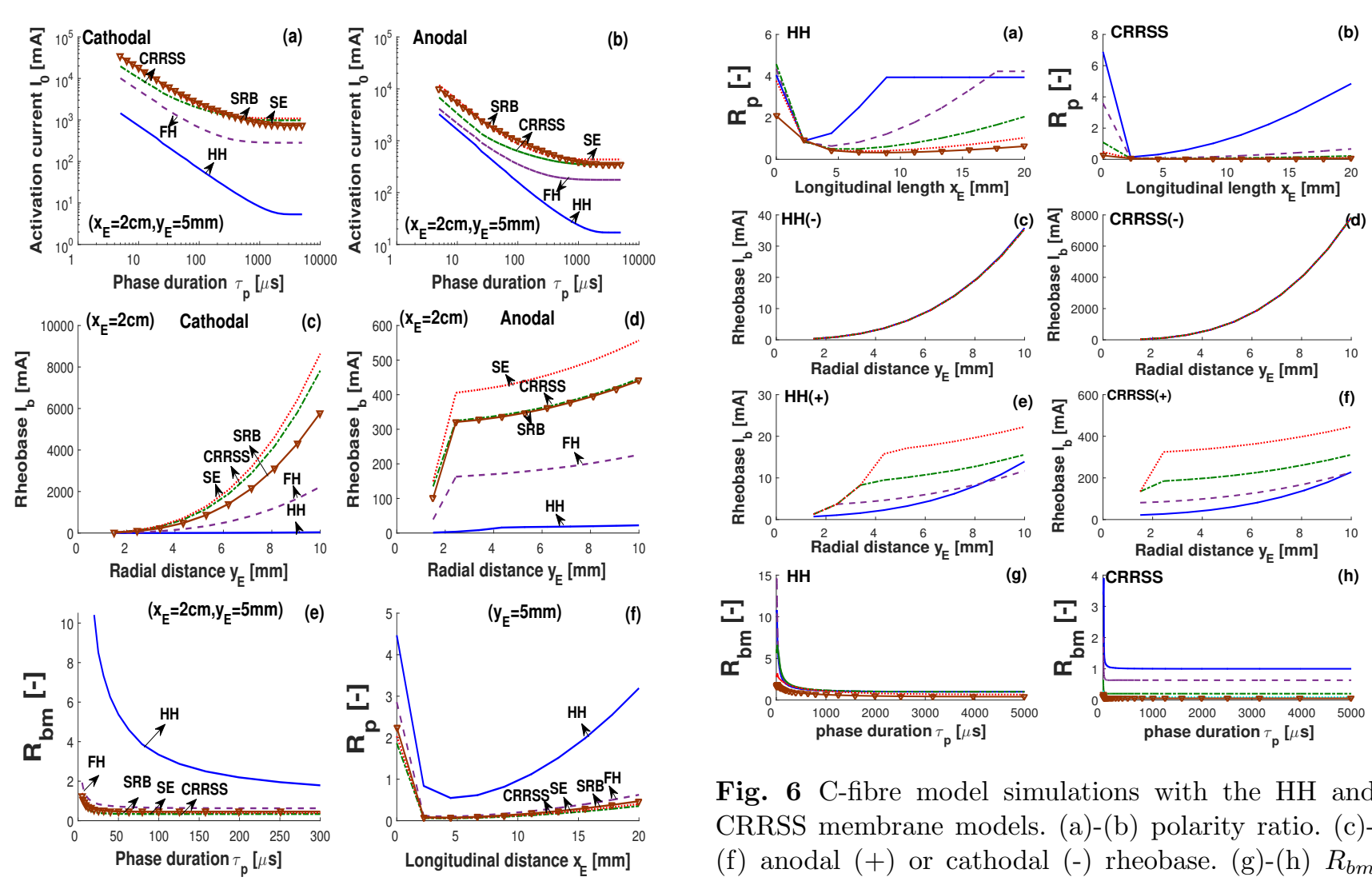

Fig. 6 C-fibre model simulations with the $\mathrm{HH}$ and CRRSS membrane models. (a)-(b) polarity ratio. (c)(f) anodal (+) or cathodal (-) rheobase. (g)-(h) $R_{b m}$ ratio. Legend. (g)-(h) (long. distance) $x_{E}=2 \mathrm{~cm}$. (a)-(b),(g)-(h) (rad. distance): (-) 2mm, (-- -) 4mm, $(-\cdots)$ ) $6 \mathrm{~mm},(\cdots \cdots) 8 \mathrm{~mm},(*) 1 \mathrm{~cm}$. (c)-(f) (long. distance): (-) $0.5 \mathrm{~cm},(---) 1 \mathrm{~cm},(-\cdots) 1.5 \mathrm{~cm},(\ldots \ldots) 2 \mathrm{~cm}$ cathodal activation current, (b) anodal activation current, (c) cathodal rheobase, (d) anodal rheobase, (e) bi/monophasic rheobase ratio (f) polarity ratio

quence of a decrease in chronaxie or strength-duration time constant when using a model with infinite myelin impedance. This decrease in time constant was theoretically predicted by Rubinstein [33].

Richardson et al. reports an increase of $\epsilon_{S D}=11 \%$ of the chronaxie when abandoning the myelin approximation, for the electrode configuration and model parameters used in their study. It can be observed from Fig. 4(a)-(b) that the error on the strength-duration time constant is dependent on the electrode position, polarity and the used membrane model. Both the error on the time constant, $\epsilon_{S D}$, as the error on the rheobase $\epsilon_{b}$, due to the myelin approximation, increase with radial electrode distance (Fig. 4). The error is furthermore determined by the mode of activation (stimulation at neural terminations or on the axon), as can be observed from Fig. 4(b).

These results show that the impact of the myelin approximation on the SD time constant and rheobase is most significant in the Frankenhaeuser-Huxley model at large electrode-neuron separations. For instance, the time constant $\tau_{e}$ is increased by $70 \%$ and the rheobase

$I_{b}$ is decreased by $-6.6 \mathrm{~mA}$ by the myelin approximation in the Frankenhaeuser-Huxley model, if the radial electrode distance is equal to $10 \mathrm{~mm}$.

\subsection{C-fibres}

Simulation results in the C-FIBRE model are shown in Fig. 5. Subsequently, Fig. 6 and Fig. 7 show more detail on the effect of the radial and longitudinal electrode position on the excitability indices in the $\mathrm{HH}$ and CRRSS models. Lower computational cost in these two conditionally linear models, allowed for more extensive parameter sweeps.

\section{Discussion}

\subsection{Strength-duration relation}

Strength-duration relations at constant electrode location, but for different membrane models, are shown in Fig. 3(a)-(b) and Fig. 5(a)-(b) for A-fibres and C-fibres 

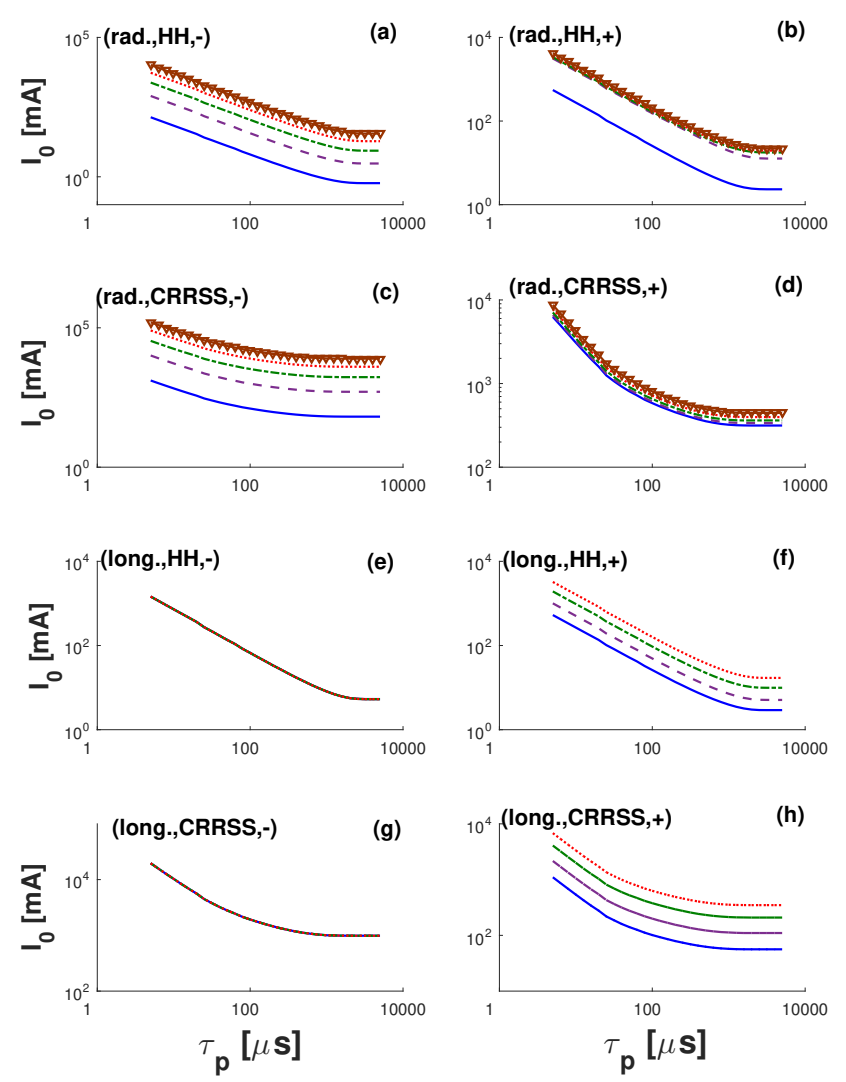

Fig. 7 C-fibre anodal (+) and cathodal (-) strengthduration plots with the $\mathrm{HH}$ and CRRSS membrane models. Legend. (a)-(d) (rad. distance): (-) 2mm, (---) 4mm, (-.-) 6mm, (…) 8mm, (*) 1cm. (long. distance): $x_{E}=20 \mathrm{~mm}$. (e)-(h) (radial distance): $y_{E}=$ $5 \mathrm{~mm}$. (long. distance): (-) $0.5 \mathrm{~cm},(---) 1 \mathrm{~cm},(-\cdots-)$ $1.5 \mathrm{~cm},(\cdots \cdots) 2 \mathrm{~cm}$

respectively. We observe that both A-fibres and C-fibres have a fixed order of excitability at long pulse durations. To compare the relative magnitude of stimulus current required to initiate an action potential, we introduce the rheobase ratio $(\mathrm{RR})$. The rheobase ratio of a model is defined as the ratio of the rheobase calculated

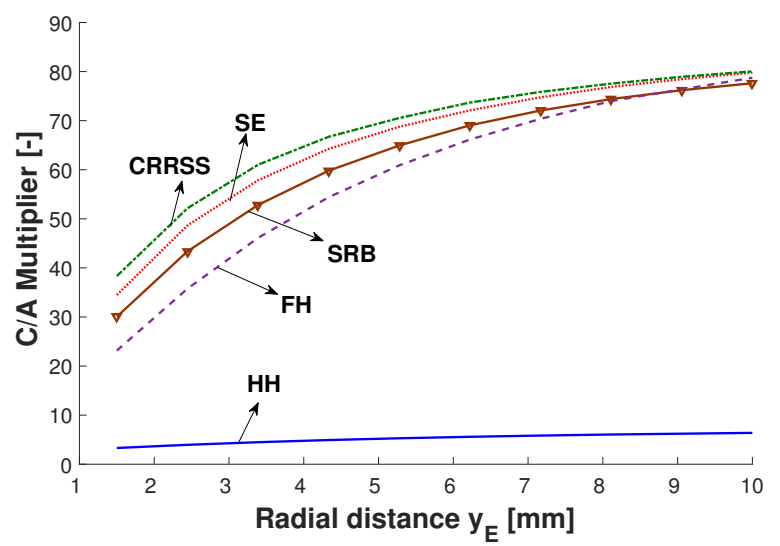

Fig. 8 Dependence of rheobase C/A multiplier on radial distance and membrane dynamics. The cathode is placed at $x_{E}=20 \mathrm{~mm}$

by the model to the rheobase that would be obtained if the membrane model would be substituted by the Frankenhaeuser-Huxley system. Consequentially, RRvalues obtained by the FH-system are equal to unity. An overview of RR-values is presented in Table 5. Models with RR-values greater than unity are more difficult to excite than the FH system at long pulse durations, while models with RR-values smaller than unity are more easy to excite.

The relative magnitude of required threshold current at long pulse durations to initiate an action potential, as compared to the Frankenhaeuser-Huxley system, is dependent on the electrode position, the electrode polarity and the type of multi-compartmental electrostimulation model (A-fibre or C-fibre). However, as can be seen from the values in Table 5, this dependency is not strong enough to change the order of excitability of the different models of membrane channel dynamics. In order of increasing threshold, we find: $\mathrm{HH}$, FH, SRB, CRRSS and SE. E.g., for cathodal stimulation of A-fibres, we find average RR-values of $0.22,1$, 2.40, 2.94 and 3.35 for the different membrane models

\begin{tabular}{|l|l|l|l|l|}
\hline Membrane & Model & Min. RR & Max. RR & Average RR \\
\hline \multirow{2}{*}{$\mathrm{HH}$} & A-fibre & $(-) 0.20 ;(+) 0.06$ & $(-) 0.27 ;(+) 0.25$ & $(-) 0.22 ;(+) 0.14$ \\
\cline { 2 - 5 } & C-fibre & $(-) 0.02 ;(+) 0.02$ & $(-) 0.04 ;(+) 0.10$ & $(-) 0.02 ;(+) 0.08$ \\
\hline \multirow{2}{*}{ CRRSS } & A-fibre & $(-) 2.00 ;(+) 1.84$ & $(-) 3.46 ;(+) 2.66$ & $(-) 2.94 ;(+) 2.26$ \\
\cline { 2 - 5 } & C-fibre & $(-) 3.31 ;(+) 1.97$ & $(-) 3.52 ;(+) 3.44$ & $(-) 3.47 ;(+) 2.13$ \\
\hline \multirow{2}{*}{ SE } & A-fibre & $(-) 2.48 ;(+) 2.30$ & $(-) 3.83 ;(+) 3.09$ & $(-) 3.35 ;(+) 2.75$ \\
\cline { 2 - 5 } & C-fibre & $(-) 3.69 ;(+) 2.46$ & $(-) 3.88 ;(+) 3.80$ & $(-) 3.84 ;(+) 2.60$ \\
\hline \multirow{2}{*}{ SRB } & A-fibre & $(-) 1.94 ;(+) 1.78$ & $(-) 2.64 ;(+) 2.23$ & $(-) 2.40 ;(+) 2.10$ \\
\cline { 2 - 5 } & C-fibre & $(-) 2.52 ;(+) 1.95$ & $(-) 2.60 ;(+) 2.60$ & $(-) 2.60 ;(+) 2.02$ \\
\hline
\end{tabular}

Table 5 Minimum, maximum and average rheobase ratio (RR) obtained by the different models for cathodal $(-)$ and anodal $(+)$ stimulation. RR-values are defined by taking the FH-system as reference. A-fibre values are calculated with the SENN-MA model 
in the aforementioned order of excitation. This order of excitability is reported as well by Rattay and Aberham [27], excluding the SRB-system, for a pulse duration of $100 \mu \mathrm{s}$ and for current directly injected into a single compartmental neuron. However, the order of excitability among the different membrane models is altered at short pulse durations, as the SRB-model becomes relatively more difficult to excite (Fig. 3 and Fig. 5).

For cathodal stimulation (Fig. 3(c) and Fig. 5(c)), rheobase ratios first diverge from unity with increasing radial electrode distance, after which they level off. For anodal stimulation (Fig. 3(d) and Fig. 5(d)), rheobase ratios reach a steady level when stimulation at the neuron terminations becomes the dominant mode of excitation.

The impact on threshold current of the presence of internodes is shown in Fig. 8. Here, the $C / A$-multiplier is the ratio of the rheobase obtained by the C-FIBRE model and the SENN-MA model. The $C / A$-multiplier values obtained by the Hodgkin-Huxley model are not in line with the other models, because the HH parameters are altered in the SENN-model (see section 2). $C / A$-multiplier values increase with electrode-neuron separation. At the largest simulated radial electrode distance, the FH, CRRSS, SE, and SRB model have similar $C / A$-multiplier values between 77 and 80 .

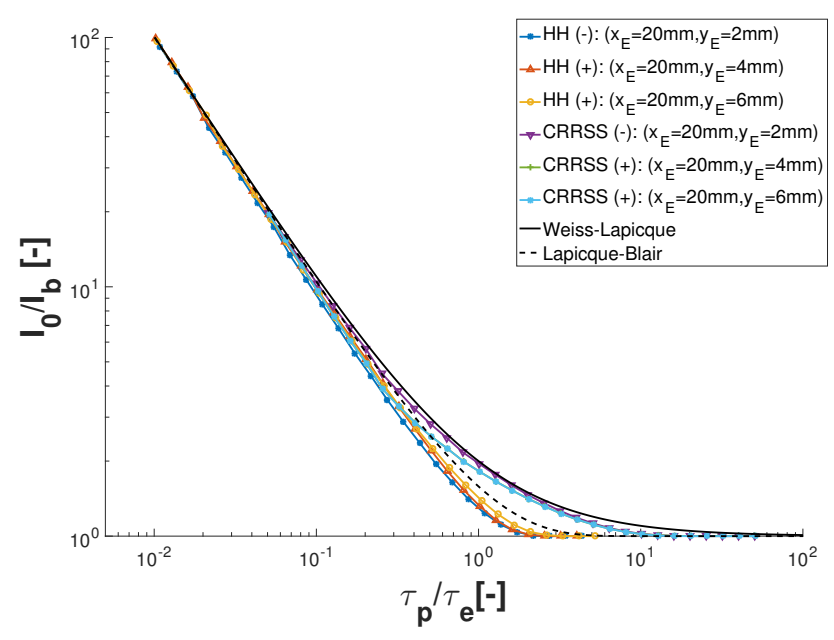

Fig. 9 Normalized SD-relations in the Hodgkin-Huxley and CRRSS models for three generic electrode locations. Cathodal (-) stimulation at a radial distance of $2 \mathrm{~mm}$, represents the case in which the neuron is stimulated by the gradient of the electric field. Anodal (+) stimulation at a radial distance of $6 \mathrm{~mm}$ represents the case in which stimulation at neuronal terminations is dominant. Anodal $(+)$ stimulation at $y_{E}=4 \mathrm{~mm}$ is shown to illustrate an intermediate case
Furthermore, the strength-duration relation was obtained for the Hodgkin-Huxley and CRRSS models, while performing extensive parameter sweeps of the electrode location and waveform polarity. The result is shown in Fig. 7, and allows us to determine the dependency of the SD-relation on the electrode and stimulus parameters. Fig. 7(a),(c) show the dependence of the SDplots on the radial cathode distance $y_{E}$ in the HodgkinHuxley and CRRSS models respectively. Increasing the cathode-neuron separation, will shift the SD-plot to higher values. Similarly, Fig 7(b)-(d) shows the impact of anode-neuron separation on SD-plots. Here, increasing the anode-neuron separation will lead to a transition from the SGE-mode to the NT-mode of activation. Consequentially, the strength-duration plot does not depend on the anode-neuron separation, for $y>y_{c}$. Here, $y_{c}$ is the critical distance, at which the transition from SGE-mode to NT-mode of activation occurs. In Fig. 7(e)-(h) it is shown that the longitudinal electrode distance will only impact the strength-duration plot for anodal stimulation. In this case, smaller anode-terminal separations result in lower threshold current values.

Most of the dependency of the SD-plots in Fig. 7 on electrode location and polarity, can be explained through the rheobase current $I_{b}$ and the SD time constant $\tau_{e}$. Indeed, both the rheobase and the time constant do depend on the electrode location and waveform polarity. Furthermore, it is possible to normalize the threshold current and pulse duration by using these excitability indices (rheobase and SD time constant). Subsequently plotting the $\frac{I_{0}}{I_{b}}\left(\frac{\tau_{p}}{\tau_{e}}\right)$-relation reveals that all normalized SD-plots coincide, under the constraint of identical membrane dynamics and dominant mode of excitation (results not shown). Indeed, it is observed that the shape of the normalized strength-duration relation is altered, when stimulation at neuronal terminations becomes the dominant mode of excitation. This is an extension of a result obtained by Šarolić et al.: it was determined by Šarolić et al. that the normalized strength-duration curve does not depend on the electrode location and spatial waveform (wire electrode, bipolar electrode setup or homogeneous E-field) in the SENN-model [34]. However, the effect of neuronal terminations, waveform polarity, and membrane dynamics was not yet taken into account.

The observation that, of the studied independent parameters, normalized SD-plots are only dependent on membrane dynamics and the dominant mode of excitation, is illustrated in Fig. 9. For both the $\mathrm{HH}$ and the CRRSS models three generic electrode configurations are used. First, a cathode at a radial distance $y_{E}=2 \mathrm{~mm}$ is used to represent stimulation by the spatial gradient of the electric field (SGE-mode). Second, 


\begin{tabular}{|l|c|c|c|c|}
\hline \multirow{2}{*}{ Membrane } & \multicolumn{2}{|c|}{ Weiss-Lapicque } & \multicolumn{2}{c|}{ Lapicque-Blair } \\
\cline { 2 - 5 } & A-fibres & C-fibre & A-fibre & C-fibre \\
\hline HH & 14.5863 & 93.3663 & 2.4681 & 56.3922 \\
\hline FH & 3.8529 & 8.05448 & 1.0775 & 0.93236 \\
\hline CRRSS & 3.5515 & 3.31010 & 1.8848 & 1.57979 \\
\hline SE & 5.1951 & 7.20470 & 0.6878 & 0.76276 \\
\hline SRB & 2.8468 & 2.33073 & 8.1539 & 3.78514 \\
\hline
\end{tabular}

Table 6 Sum of squares of residuals $\left(S S_{r e s}[-]\right)$. A-fibre values are calculated with the SENN-MA model

an anode at $y_{E}=6 \mathrm{~mm}$ is used, to illustrate stimulation at neuronal terminations (NT-mode). It is indeed clear from Fig. 9 that normalized strength-duration curves do depend on the dominant mode of excitation, as well as the membrane dynamics. An intermediate case is represented by an anode at a radial distance $y_{E}=$ $4 \mathrm{~mm}$. For the Hodgkin-Huxley model, the transition from the SGE-mode to the NT-mode occurs at anodal radial distances of $y_{E}=4.33 \mathrm{~mm}( \pm 0.95 \mathrm{~mm})$ in the used C-FIBRE model. As a consequence, an intermediate strength-duration curve is observed, indicating a continuous transition of the normalized SD-curve with increasing radial distance. In contrast, this transition between dominant modes of excitation is observed at a radial anodal distance of $2.44 \mathrm{~mm}( \pm 0.95 \mathrm{~mm})$ for all other membrane models (see also the subsequent subsection). Consequentially, the normalized SD-plots for $y_{E}=4 \mathrm{~mm}$ and $y_{E}=6 \mathrm{~mm}$ coincide in the CRRSSmodel.

The strength-duration relations, obtained with the different membrane models and by excitation wihin the
SGE-mode, are compared with the Weiss-Lapicque and Lapicque-Blair equations in Fig. 10. Furthermore, sum of squares of residuals $\left(S S_{\text {res }}\right)$ values are summarized in Table 6. It is observed, that fitting the SD-relation by the Lapicque-Blair equation is superior for the $\mathrm{HH}, \mathrm{FH}$, CRRSS and SE models. Conversely, SD-plots obtained by the SRB-model within the SGE-mode of excitation, are better approximated by the Weiss-Lapicque equation.

\subsection{Dependence on fibre diameter}

The dependence of the rheobase current and SD time constant on the fibre diameter in the C-FIBRE model is shown in Fig. 11(a)-(b). In Fig. 11(a) the rheobase multiplier $M=I_{b}(D) / I_{b}(D=10 \mu m)$ is shown for diameters between $1 \mu \mathrm{m}$ and $10 \mu \mathrm{m}$. Rheobase current is inversely proportional with fibre diameter. The slope of the $\log M-\log D$ plot fluctuates around -1 in the FH, CRRSS, SE, and SRB model (min: -1.1, max: -0.9 ), indicating that approximately $I_{b} \propto 1 / D$. In the
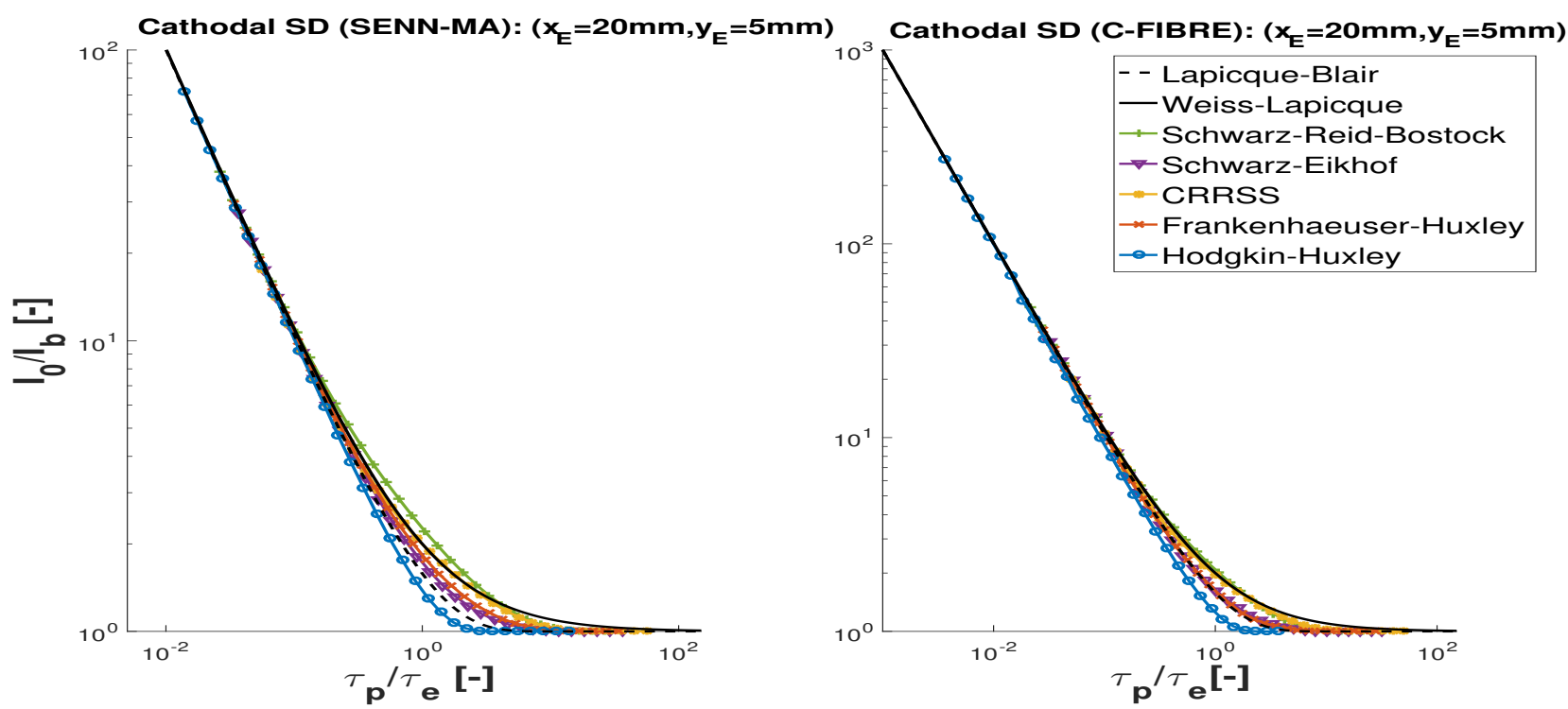

Fig. 10 Correspondence of normalized strength-duration plots with the Weiss-Lapicque and Lapicque-Blair relations. Electrode location $\left(x_{E}=2 \mathrm{~cm}, y_{E}=5 \mathrm{~mm}\right)$ and polarity (cathodal) is chosen to ensure that neuronal excitation is caused by the spatial gradient of the electric field at the centre of the axon 
Hodgkin-Huxley model the slope in the $\log M-\log D$ plot lies between -0.95 and -0.8 for $x_{E}=20 \mathrm{~mm}$ and $y_{E}=5 \mathrm{~mm}$ (Fig. 11(a)). Fig. 11(c) investigates the role of cathode-neuron separation on the rheobase-diameter relation. In the CRRSS model, a $I_{b} \propto 1 / D$ relation is found between rheobase and fibre diameter, for all simulated radial distances $1.5 \mathrm{~cm} \leq y_{E} \leq 10 \mathrm{~cm}$. It is observed from Fig. 11(c) that the $I_{b} \propto 1 / D$ relation will be valid for the Hodgkin-Huxley model as well, for large cathode-neuron separations. We conclude that for C-fibres, a $I_{b} \propto 1 / D$ relation holds for large radial electrode distances and that the Hodgkin-Huxley model requires a larger radial distance than the other simulated models. This is in contrast with A-fibres models, for which the slope in the $\log M-\log D$ plot decreases with increasing diameter (McNeal [22]).

Similarly, Fig. 11(b) shows that the SD time constant $\tau_{e}$ is approximately constant over the simulated diameter range in the $\mathrm{FH}, \mathrm{CRRSS}, \mathrm{SE}$, and SRB model. In the Hodgkin-Huxley model, the time constant decreases with diameter. In Fig 11(d) it is shown that the sensitivity of the time constant on the fibre diameter is reduced at large cathode-neuron separations.

\subsection{Polarity ratio}

If the neuron is excited by the spatial gradient of the electric field rather than at the neuronal terminations, then cathodal stimulation is more effective than anodal stimulation and $R_{p}>1$. However, if the radial electrode distance $y_{E}$ is increased or the longitudinal electrode distance $x_{E}$ is decreased, stimulation at the neuronal terminations becomes the dominant mode of excitation. This is expressed by a decrease in the polarity ratio $R_{p}$.

The polarity ratio is shown for A-fibres and C-fibres in Fig. 3(f) and Fig. 5(f) respectively at $\left(x_{E}, y_{E}\right)=$ $(2 \mathrm{~cm}, 5 \mathrm{~mm})$. The polarity ratio is furthermore shown for different radial and longitudinal electrode positions in the C-FIBRE model in Fig. 6(a)-(b). These results show that the polarity ratio drops as the location of the virtual electrode approaches the neural terminal. Furthermore, it can be observed from Fig. 6(a)-(b) that the minimum in the $R_{p}\left(x_{E}\right)$ plots shifts to higher values of $x_{E}$, for greater radial electrode distances.

The transition from the SGE-mode to the NT-mode of excitation occurs when the polarity ratio $R_{p}$ begins to decrease. This transition is dependent on the used membrane model. For example, consider an electrode at a longitudinal distance $x_{E}=2 \mathrm{~cm}$ of an unmyelinated fibre. We investigate the critical radial distance of the electrode $y_{c}$ for which the transition between these two modes of excitation occurs. In Fig. 6(a), different radial distances $(2 \mathrm{~mm}, 4 \mathrm{~mm}, 6 \mathrm{~mm}, 8 \mathrm{~mm}$ and
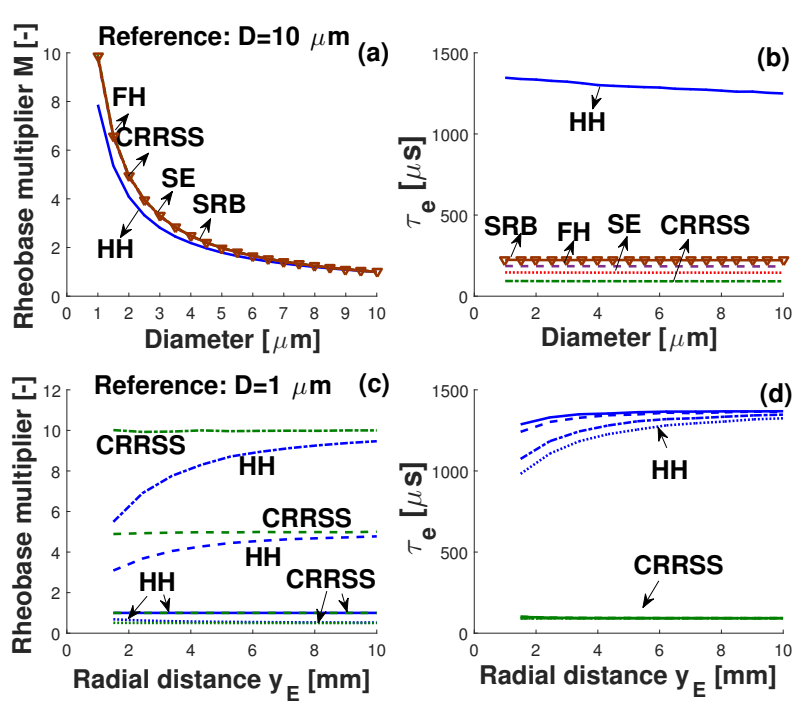

Fig. 11 Impact of diameter on rheobase $((a),(c))$ and SD time constant ((b),(d)) in C-FIBRE. (a) Rheobase multipliers (reference indicated in figure) and SD time constants are plotted against diameter ((a)-(b)) and radial electrode distance $((\mathrm{c})-(\mathrm{d}))$. Cathode location is $\left(x_{E}=20 \mathrm{~mm}, y_{E}=5 \mathrm{~mm}\right)$ in (a)-(b) and $x_{E}=20 \mathrm{~mm}$ in (c)-(d). Legend. (diameter) (c) (….) $0.5 \mu \mathrm{m},(---)$ $5 \mu \mathrm{m},(-\cdots) 10 \mu \mathrm{m}$. (d) (diameter): (-) $0.5 \mu \mathrm{m},(---)$ $1 \mu \mathrm{m},(-\cdots) 5 \mu \mathrm{m},(\cdots \cdots) 10 \mu \mathrm{m}$. Method. Plot was obtained by first order Crank-Nicolson discretisation with time step $\Delta t=\min \left(\tau_{p} / 50,0.01 \mathrm{~ms}\right)$

$1 \mathrm{~cm}$ ) are represented by five curves. The dependency of the polarity ratio on the radial distance $y_{E}$ is estimated by comparing the values indicated by the five curves at the constant longitudinal distance $x_{E}=2 \mathrm{~cm}$. We observe that for the Hodgkin-Huxley membrane model, the polarity ratio starts to decrease at a radial distance between $4 \mathrm{~mm}$ and $6 \mathrm{~mm}$. In contrast, in the CRRSS model (Fig. 6(b)) the critical distance lies between $2 \mathrm{~mm}$ and $4 \mathrm{~mm}$. Another procedure to determine the critical radial electrode distance is by investigating for the discontinuity in the slope of anodal rheobasedistance curves, as will be described below.

\section{$4.4 \mathrm{Bi} /$ monophasic ratio}

If cathodal stimulation is more effective than anodal stimulation in initiating an action potential $\left(R_{p}>1\right)$, then the bi/monophasic ratio $R_{b m}$ will always be larger than one. This is the case for the SENN-M and SENNMA models at $\left(x_{E}, y_{E}\right)=(2 \mathrm{~cm}, 5 \mathrm{~mm})$, see Fig. $3(\mathrm{e})$. If the phase duration $\tau_{p}$ is less than the strength-duration time constant $\tau_{e}, R_{b m}$-values are significantly greater than unity. This is due to the fact that the reversed cur- 
rent in the second phase of the biphasic pulse, can stop the development of the action potential ([29], pp 13241). For long pulse durations however, the two phases of the waveform do not negate each other's influence, and $R_{b m}=1$ [28].

This reasoning does not hold true if $R_{p}<1$, see Fig. 6(g)-(h). It is now possible that the first (cathodal) phase is not able to initiate an AP, while the subsequent (anodal) phase is. Consequentially, in this case biphasic stimulation would be more effective than monophasic stimulation, resulting in $R_{b m}<1$.

4.5 Rheobase-distance relation and critical electrode distance

The critical radial electrode distance $y_{c}$ for the transition between the SGE-mode and NT-mode of excitation, can be obtained from the anodal rheobase-distance (RD) relations (Fig. 3(d), Fig. 5(d) and Fig. 6(e)-(f)). This critical distance $y_{c}$ is the abscissa, associated with the "kink" in the anodal RD-curve. In contrast, cathodal RD-relations are smooth and do not exhibit a kink (Fig. 3(c), Fig. 5(c), Fig. 6(c)-(d)). The discontinuity in the spatial derivative w.r.t. $y_{E}$ of the anodal RD-curve at the critical electrode distance $y_{c}$ is a consequence of the polarity ratio $R_{p}$, that starts to decrease at $y_{c}$. Indeed, the anodal rheobase $I_{b}^{(+)}$can be expressed as a function of $R_{p}$ and the cathodal rheobase $I_{b}^{(-)}$:

$I_{b}^{(+)}\left(y_{E}\right)=R_{p}\left(y_{E}\right) I_{b}^{(-)}\left(y_{E}\right)$

The derivative of $I_{b}^{(-)}\left(y_{E}\right)$ does not exhibit a discontinuity. Consequentially, the "kink" in the anodal RDcurve is related with a discontinuity in slope in the $R_{p}\left(y_{E}\right)$-curve, i.e. when the polarity ratio drops at the critical distance $y_{c}$.

As an example, the critical radial distance $y_{c}$ in $\mathrm{C}$ fibres at $x_{E}=2 \mathrm{~cm}$ is obtained by looking for the discontinuity in slope in Fig. 5(d). The uncertainty on $y_{c}$ is determined by the size of the step in the parametersweep. The critical radial distance $y_{c}$, that indicates the transition between the dominant modes of excitation for stimulation with long pulse durations, is dependent on the used membrane model. For the Hodgkin-Huxley model we find $y_{c}=4.33 \mathrm{~mm}( \pm 0.95 \mathrm{~mm})$, while all other models have $y_{c}=2.44 \mathrm{~mm}( \pm 0.95 \mathrm{~mm})$.

\section{Limitations}

The goal of this study is to compare excitability in different models of spatially extended neurons, for a variety of stimulus and electrode parameters. To this end, simulations are restricted to three different representations of a multi-compartmental axon (SENNMA, SENN-M and C-FIBRE). A fourth axonal model (MRG) was developed by Blight, and aims to unravel the mechanism for depolarising afterpotentials [2]. Only the MRG-model has the important feature of reproducing the depolarising afterpotential (DAP) and afterhyperpolarisation (AHP). However, the DAP and AHP have no direct impact on the excitability indices, which are determined by the first depolarisation in the action potential. Nevertheless, excitability is still influenced by the double cable structure. Several researchers have used similar models in their computational studies [37, 32, 21]. However, these double-cable models are more computationally expensive than the used SENN and C-FIBRE model. Because of this reason, the impact of double-cable structures and explicit modeling of the paranodes and juxtaparanodes on excitability indices was not included in this study.

Similarly, several specialized models of the active membrane have been proposed in literature. E.g., at present, Channelopedia lists 52 different HH-type models for active currents [24]. Consequentially, we restricted this comparative study to five often used models, describing the active membrane.

Finally, a relatively low model temperature of $18.5^{\circ} \mathrm{C}$ was chosen, to prevent heat-block in the unmodified HH-equation set in the C-FIBRE model. Higher model temperatures result in faster membrane gate dynamics, leading to shorter action potentials, faster propagation of the action potential, shorter summation, and excitation time constants, ... [5]. Because temperature alters the membrane dynamics (through multiplication with a temperature dependent factor $k$ of the modeldependent rate constants $\alpha$ and $\beta$, see Eq. (11)-(10)) and because in this study it is observed that membrane channel dynamics has a significant impact on excitation threshold, a similar impact of temperature on excitation is expected. However, modulation of membrane dynamics by changing the model temperature is qualitatively similar to substituting the model of membrane dynamics. E.g., in a study by Cartee, the temperature is used to alter the membrane dynamics in order to obtain a better fit to the summation and refraction properties of the cochlear nerve [5]. However, changing the temperature will have a more modest impact on the membrane dynamics, than substituting the equation set. As a consequence of this similarity between substituting the equation set for membrane dynamics and changing the temperature, it is expected that the conclusions of our study will not be qualitatively altered by a different model temperature. For example, we observed that the strength-duration time constant is independent of the 
fibre diameter for large electrode-neuron separations. This result is valid for all studied membrane models, although larger electrode-neuron separations are necessary for the HH-model. As a consequence, it is unlikely that a different temperature would have a significant impact on this result.

\section{Conclusion}

Threshold currents, rheobase, polarity ratio, bi/monophasic ratio and SD time constants are widely used in literature to quantize neuronal excitability. In this paper, three multi-compartmental models are combined with five models of membrane dynamics to generate plots of these excitability indices for a wide range of electrode locations and stimulus properties. Excitability in different membrane and spatial models is compared by the rheobase ratio $R R$ and $C / A$-multiplier, defined in this study. Furthermore, SD-plots obtained by the different computational models are compared with Weiss-Lapicque and Lapicque-Blair fits. We summarise the following conclusions (Fig. 3, 5, 7-10, Table 5-6).

- The used membrane model has a significant impact on the observed excitation threshold. In order of increasing excitation threshold, we found average cathodal rheobase ratios of $0.22,1,2.40,2.94$ and 3.35 for the HH, FH, SRB, CRRSS and SE models respectively. E.g., this means that for an average cathode position the SE-model is 3.35 times more difficult to excite with long pulses than the FHmodel.

- The difference in excitability between the membrane models becomes more pronounced for larger separation between the electrode and the neuron, while the neuron is stimulated by the spatial gradient of the electric field (SGE-mode).

- The rheobase ratio is independent of the electrodeneuron separation for neurons stimulated at the terminations (NT-mode).

- Membrane channel dynamics determine the shape of the strength-duration plot. HH, FH, CRRSS, and SE models are better approximated by the LapicqueBlair formula, while the Weiss-Lapicque equation is a better fit for the SRB-model. Normalized SD-plots are independent of the electrode position, stimulus polarity and waveform.

- Excluding the HH-model, it is observed from comparison between the SENN-MA and C-FIBRE model that for large cathode-neuron separations C-fibre models are about 77 to 80 times more difficult to excite than A-fibre models. This $C / A$-multiplier is dependent on the membrane channel dynamics, only for short cathode-neuron separations.

The impact of stimulation at neuronal terminations is investigated. Sealed-end terminals impact anodal RD plots (resulting in a discontinuity at the critical electrode distance $y_{c}$ ), impact the bi/monophasic ratio and polarity ratio $\left(R_{b m}<1\right.$ is possible if the neuron is activated by the NT-mode) and lead to a change in the normalized SD-plots (Fig. 3-6, 9).

We compared the strength-duration and rheobasedistance plots between the SENN-M and SENN-MA models, in order to investigate the effect of the myelin approximation on neuronal excitability. Neglecting the myelin admittance always leads to a reduction in the rheobase and SD time constant. However, we found that the significance of this approximation error is strongly dependent on several interacting factors (electrode location, stimulus polarity, membrane channel dynamics, etc.). As a consequence, specification of these factors is necessary when adressing questions about the justification of the myelin approximation in a simulation study or about the effect of demyelination on neuronal excitability. In particular, we found the following (Fig. 4).

- The reduction in rheobase and SD time constant, due to the myelin approximation, is more significant for larger electrode-neuron separations

- The impact of the myelin impedance on the rheobase current is dependent on the selected membrane model. E.g., a maximal error in rheobase of $57 \%$ was found for a model with Hodgkin-Huxley dynamics. In contrast, maximal rheobase errors are smaller than $3.2 \%$ for SE or SRB models.

- The impact of the myelin impedance on the SD time constant is dependent on the selected membrane model. E.g., a maximal error in time constant of $70 \%$ and $20 \%$ is observed for a model with FH-dynamics and HH-dynamics, respectively.

- The error in strength-duration time constant $\epsilon_{S D}$ is independent of the electrode-neuron separation for neurons in which NT-mode is the dominant (i.e., for short neurons or large electrode-neurons separations).

Finally, the impact of electrode location and membrane dynamics on the $I_{b}(D)$ and $\tau_{e}(D)$ relations was investigated. In our C-FIBRE models, we observed that (Fig. 11):

- For sufficiently large electrode-neuron separations, the rheobase $I_{b}$ is inversely proportional to the fibre diameter $D$ (i.e., $\left.I_{b} \propto 1 / D\right)$. This result is independent of the membrane channel dynamics. Furthermore, this result is in contrast with A-fibre models, 
in which the slope of the $\log M-\log D$ plot decreases with increasing diameter.

- For sufficiently large electrode-neuron separations, the SD time constant $\tau_{S D}$ is independent of the fibre diameter $D$.

These proportionalities $\left(I_{b} \propto 1 / D\right.$ and $\left.\tau_{S D} \propto 1\right)$ are valid for sufficiently large electrode-neuron separations. Consequentially, deviations are found if the electrode is too close to the neuron. Larger electrode-neuron separations are required in the Hodgkin-Huxley model, as compared to the other simulated membrane models.

\section{A Summary of model equations}

Model equations and parameters are summarized in this appendix. All equations are taken from literature: references are given in section 2 . Systems of equations are discretised according to a first (SENN-M) or second (SENN-MA, C-FIBRE) order staggered Crank-Nicolson scheme (time step $\Delta t$ and spatial step $\Delta x$ ). Discretisation of the cable equation in the most general case yields:

$$
\begin{aligned}
& \frac{2 C_{m}(l)^{*}}{\Delta t}\left(V\left(l, t+\frac{\Delta t}{2}\right)-V(l, t)\right)+I_{s}\left(l, t+\frac{\Delta t}{2}\right) \\
& =G_{a, f}^{*}(l-\Delta l)\left(V\left(l-\Delta l, t+\frac{\Delta t}{2}\right)-V\left(l, t+\frac{\Delta t}{2}\right)\right)+ \\
& G_{a, b}^{*}(l+\Delta l)\left(V\left(l+\Delta l, t+\frac{\Delta t}{2}\right)-V\left(l, t+\frac{\Delta t}{2}\right)\right)+ \\
& G_{a, f}^{*}(l-\Delta l)\left(V_{e}\left(l-\Delta l, t+\frac{\Delta t}{2}\right)-V_{e}\left(l, t+\frac{\Delta t}{2}\right)\right)+ \\
& \quad G_{a, b}^{*}(l+\Delta l)\left(V_{e}\left(l+\Delta l, t+\frac{\Delta t}{2}\right)-V_{e}\left(l, t+\frac{\Delta t}{2}\right)\right)
\end{aligned}
$$

Here $C_{m}(l)^{*}$ is the membrane capacitance of the compartment at $x=l$. It is obtained from the capacitance per unit area $c_{m}$, which is $0.0073 \mu \mathrm{F} / \mathrm{cm}^{2}, 1 \mu \mathrm{F} / \mathrm{cm}^{2}, 2 \mu \mathrm{F} / \mathrm{cm}^{2}, 2.5$ $\mu \mathrm{F} / \mathrm{cm}^{2}, 2.8 \mu \mathrm{F} / \mathrm{cm}^{2}$, and $2.8 \mu \mathrm{F} / \mathrm{cm}^{2}$ for the myelinated internodes, HH, FH, CRRSS, SE, and SRB model respectively. $G_{a, b}^{*}(l)$ is the "backward" axial conductance between the compartments at $x=l-\Delta l$ and $x=l$. Similarly, $G_{a, f}^{*}(l)$ is the "forward" axial conductance between the compartments at $x=l$ and $x=l+\Delta l . V(l, t)$ is the transmembranepotential, while $V_{e}$ stands for the external potential. Finally, $I_{s}$ is the ionic membrane current. The external potential is calculated analytically:

$V_{e}=\frac{\rho_{e} I}{4 \pi r}$

Here, $\rho_{e}=3 \Omega m$ is the extracellular resistivity and $r$ is the distance from the electrode. The membrane voltage at $V(t+$ $\Delta t)$ is then calculated by:

$V(t+\Delta t)=2 V\left(t+\frac{\Delta t}{2}\right)-V(t)$

The sodium gate parameter is progressed in time by:

$$
\begin{aligned}
& \frac{m\left(t+\frac{3}{2} \Delta t\right)-m\left(t+\frac{1}{2} \Delta t\right)}{\Delta t} \\
& =k\left[\alpha_{m}(t+\Delta t)\left(1-\frac{m\left(t+\frac{3}{2} \Delta t\right)+m\left(t+\frac{1}{2} \Delta t\right)}{2}\right)\right. \\
& \left.\quad-\beta_{m}(t+\Delta t) \frac{m\left(t+\frac{3}{2} \Delta t\right)+m\left(t+\frac{1}{2} \Delta t\right)}{2}\right]
\end{aligned}
$$

And similar expressions for the other gate parameters $n, h$, and $p . \alpha$ and $\beta$ are model specific rate constants, that depend non-linearly on transmembranevoltage $V$. Finally, $k$ is a temperature constant, given by:

$k=Q_{10}^{\left(T-T_{0}\right) / 10}$

The temperature $T_{0}$ is $6.3^{\circ} \mathrm{C}$ for the $\mathrm{HH}$-model, $20^{\circ} \mathrm{C}$ for the FH-model, and $37^{\circ} \mathrm{C}$ for the other membrane models. The temperature constant $Q_{10}=3$, except for:

$Q_{10, F H}\left(\alpha_{m}\right)=1.8 ; Q_{10, F H}\left(\alpha_{n}\right)=3.2 ; Q_{10, F H}\left(\alpha_{h}\right)=2.8$

$Q_{10, F H}\left(\beta_{m}\right)=1.7 ; Q_{10, F H}\left(\beta_{n}\right)=2.8 ; Q_{10, F H}\left(\beta_{h}\right)=2.9$

$Q_{10, S E}(m)=2.2 ; Q_{10, S E}(h)=2.9$

$Q_{10, S R B}(m)=2.2 ; Q_{10, S R B}(h)=2.9$

Here $Q_{10, I}(j)$ refers to the temperature coefficient for $\alpha_{j}$ and $\beta_{j}$ of membrane model $I$.

Previous equations can be cast in two sets of matrix equations, in the case of the conditionally linear membrane models (HH and CRRSS models). Consequentially, a second order correct solution is obtained without iteration for the HodgkinHuxley and CRRSS model. The other models (FH, SE, and SRB) are not conditionally linear and iteration of the equations is necessary, to obtain a solution that is correct to second order in the discretisation time.

Each model of membrane channel dynamics contains a loss current expressed by:

$i_{L}=g_{L}\left(V-V_{L}\right)$

Here, $g_{L}$ is the leak conductance and is equal to $0.015 \mathrm{mS} / \mathrm{cm}^{2}$, $0.3 \mathrm{mS} / \mathrm{cm}^{2}, 30.3 \mathrm{mS} / \mathrm{cm}^{2}, 128 \mathrm{mS} / \mathrm{cm}^{2}, 86 \mathrm{mS} / \mathrm{cm}^{2}$, and 60 $\mathrm{mS} / \mathrm{cm}^{2}$ for the myelinated internodes, HH, FH, CRRSS, SE, and SRB model respectively. Furthermore, the bias voltage $V_{L}$ is $-59.4 \mathrm{mV},-69.974 \mathrm{mV},-80.01 \mathrm{mV},-78 \mathrm{mV},-84 \mathrm{mV}$ for the HH, FH, CRRSS, SE, and SRB model respectively. An example of the spatiotemporal response, generated by the used membrane models, is shown in Fig. 12.

\section{A.1 Hodgkin-Huxley model}

The ionic membrane current $I_{s}$ is related to the ionic membrane current per area, by the expression $i_{s} \pi d \Delta l=I_{s}$. The Hodgkin-Huxley model consists of a natrium current $i_{N a}$, potassium current $i_{K}$, and a loss current $i_{L}$ :

$i_{N a}=g_{N a} m^{3} h\left(V-V_{N a}\right)$

$i_{K}=g_{K} n^{4}\left(V-V_{K}\right)$

$g_{K}=36 \mathrm{mS} / \mathrm{cm}^{2}$ and $g_{N a}=120 \mathrm{mS} / \mathrm{cm}^{2}$ are maximal conductances per area. $V_{N a}=45 \mathrm{mV}$ and $V_{K}=-82 \mathrm{mV}$ are Nernst-potentials for sodium and potassium respectively. The Hodgkin-Huxley rest potential is $V_{r}=-70 \mathrm{mV}$.

The rate coefficients are given by (with $V$ in millivolts):

$\tilde{V}=V-V_{r}$

$\alpha_{m}=\frac{2.5-0.1 \tilde{V}}{\exp (2.5-0.1 \tilde{V})-1} ; \beta_{m}=4 \exp \left(-\frac{\tilde{V}}{18}\right)$

$\alpha_{n}=\frac{0.1-0.01 \tilde{V}}{\exp (1-0.1 \tilde{V})-1} ; \beta_{n}=0.125 \exp \left(-\frac{\tilde{V}}{80}\right)$

$\alpha_{h}=0.07 \exp \left(-\frac{\tilde{V}}{20}\right) ; \beta_{h}=(\exp (3-0.1 \tilde{V})+1)^{-1}$ 
A.2 Chiu-Ritchie-Rogert-Stagg-Sweeney model

The CRRSS model consists of a sodium and loss current:

$i_{s}=g_{N a} m^{2} h\left(V-V_{N a}\right)+g_{L}\left(V-V_{L}\right)$

Here, $g_{N a}=1445 \mathrm{mS} / \mathrm{cm}^{2}, V_{N a}=35 \mathrm{mV}$, and $V_{r}=-80 \mathrm{mV}$.

The equations for the rate coefficients are:

$\alpha_{m}=\frac{97+0.363 \tilde{V}}{1+\exp \left(\frac{31-\tilde{V}}{5.3}\right)} ; \beta_{h}=\frac{15.6}{1+\exp \left(\frac{24-\tilde{V}}{10}\right)}$

$\alpha_{h}=\frac{15.6}{\left[1+\exp \left(\frac{24-\tilde{V}}{10}\right)\right]\left[\exp \left(\frac{\tilde{V}-5.5}{5}\right)\right]}$

$\beta_{m}=\frac{97+0.363 \tilde{V}}{\left[1+\exp \left(\frac{31-\tilde{V}}{5.3}\right)\right]\left[\exp \left(\frac{\tilde{V}-23.8}{4.17}\right)\right]}$

\section{A.3 Frankenhaeuser-Huxley model}

The Frankenhaeuser-Huxley model contains a sodium, potassium, non-specific and loss current. The rest potential is $V_{r}=$ $-70 \mathrm{mV}$.

$i_{N a}=P_{N a} m^{2} h \frac{V F^{2}}{R T} \frac{[N a]_{0}-[N a]_{i} \exp \frac{V F}{R T}}{1-\exp \frac{V F}{R T}}$

$i_{K}=P_{K} n^{2} \frac{V F^{2}}{R T} \frac{[K]_{0}-[K]_{i} \exp \frac{V F}{R T}}{1-\exp \frac{V F}{R T}}$

$i_{p}=P_{p} p^{2} \frac{V F^{2}}{R T} \frac{[N a]_{0}-[N a]_{i} \exp \frac{V F}{R T}}{1-\exp \frac{V F}{R T}}$

$\alpha_{m}=\frac{0.36(\tilde{V}-22)}{1-\exp \left(\frac{22-\tilde{V}}{3}\right)} ; \beta_{m}=\frac{0.4(13-\tilde{V})}{1-\exp \left(\frac{\tilde{V}-13}{20}\right)}$

$\alpha_{n}=\frac{0.02(\tilde{V}-35)}{1-\exp \left(\frac{35-\tilde{V}}{10}\right)} ; \beta_{n}=\frac{0.05(10-\tilde{V})}{1-\exp \left(\frac{\tilde{V}-10}{10}\right)}$

$\alpha_{h}=-\frac{0.1(\tilde{V}+10)}{1-\exp \left(\frac{\tilde{V}+10}{6}\right)} ; \beta_{h}=\frac{4.5}{1+\exp \left(\frac{45-\tilde{V}}{10}\right)}$

$\alpha_{p}=\frac{0.006(\tilde{V}-40)}{1-\exp \left(\frac{-\tilde{V}+40}{10}\right)} ; \beta_{p}=-\frac{0.09(\tilde{V}+25)}{1-\exp \left(\frac{\tilde{V}+25}{20}\right)}$

Here, the constants are:

$P_{n a}=0.008 \mathrm{~cm} / \mathrm{s} ; P_{K}=0.0012 \mathrm{~cm} / \mathrm{s} ; P_{P}=0.00054 \mathrm{~cm} / \mathrm{s}$

$[N a]_{0}=114.5 \mathrm{mmol} / \mathrm{l} ;[N a]_{i}=13.7 \mathrm{mmol} / 1$

$[K]_{0}=2.5 \mathrm{mmol} / \mathrm{l} ;[K]_{i}=120 \mathrm{mmol} / \mathrm{l}$
A.4 Schwarz-Eikhof model

The SE-model constains a sodium, potassium and loss current:

$i_{N a}=P_{N a} m^{3} h \frac{V F^{2}}{R T} \frac{[N a]_{0}-[N a]_{i} \exp \frac{V F}{R T}}{1-\exp \frac{V F}{R T}}$

$i_{K}=P_{K} n^{2} \frac{V F^{2}}{R T} \frac{[K]_{0}-[K]_{i} \exp \frac{V F}{R T}}{1-\exp \frac{V F}{R T}}$

$\alpha_{m}=\frac{1.87(\tilde{V}-25.41)}{1-\exp \left(\frac{25.41-\tilde{V}}{6.06}\right)} ; \beta_{m}=\frac{3.97(21-\tilde{V})}{1-\exp \left(\frac{\tilde{V}-21}{9.41}\right)}$

$\alpha_{n}=\frac{0.13(\tilde{V}-35)}{1-\exp \left(\frac{35-\tilde{V}}{10}\right)} ; \beta_{n}=\frac{0.32(10-\tilde{V})}{1-\exp \left(\frac{\tilde{V}-10}{10}\right)}$

$\alpha_{h}=-\frac{0.55(\tilde{V}+27.74)}{1-\exp \left(\frac{\tilde{V}+27.74}{9.06}\right)} ; \beta_{h}=\frac{22.6}{1+\exp \left(\frac{56-\tilde{V}}{12.5}\right)}$

The rest potential of the SE-model is $V_{r}=-78 \mathrm{mV}$. The other constants are:

$P_{n a}=0.00328 \mathrm{~cm} / \mathrm{s} ; P_{K}=0.000134 \mathrm{~cm} / \mathrm{s}$

$[N a]_{0}=154 \mathrm{mmol} / \mathrm{l} ;[N a]_{i}=8.71 \mathrm{mmol} / 1$

$[K]_{0}=5.9 \mathrm{mmol} / \mathrm{l} ;[K]_{i}=155 \mathrm{mmol} / \mathrm{l}$

A.5 Schwarz-Reid-Bostock model

The SRB-model contains a sodium, fast and slow potassium and loss current:

$i_{N a}=P_{N a} m^{3} h \frac{V F^{2}}{R T} \frac{[N a]_{0}-[N a]_{i} \exp \frac{V F}{R T}}{1-\exp \frac{V F}{R T}}$

$i_{K, f}=g_{K, f} n^{4}\left(V-V_{K}\right)$

$i_{K, s}=g_{K, s} p\left(V-V_{K}\right)$

Here, $g_{K, f}=30 \mathrm{mS} / \mathrm{cm}^{2}, g_{K, s}=60 \mathrm{mS} / \mathrm{cm}^{2}, V_{K}=-84 \mathrm{mV}$ and $V_{r}=-84 \mathrm{mV}$.

$\alpha_{m}=\frac{4.6(\tilde{V}-65.6)}{1-\exp \left(\frac{-\tilde{V}+65.6}{10.3}\right)} ; \beta_{m}=\frac{0.33(61.3-\tilde{V})}{1-\exp \left(\frac{\tilde{V}-61.3}{9.16}\right)}$

$\alpha_{n}=\frac{0.0517(\tilde{V}+9.2)}{1-\exp \left(\frac{-\tilde{V}-9.2}{1.1}\right)} ; \beta_{n}=\frac{0.092(8-\tilde{V})}{1-\exp \left(\frac{\tilde{V}-8}{10.5}\right)}$

$\alpha_{h}=\frac{-0.21(\tilde{V}+27)}{1-\exp \left(\frac{\tilde{V}+27}{11}\right)} ; \beta_{h}=\frac{14.1}{1+\exp \left(\frac{55.2-\tilde{V}}{13.4}\right)}$

$\alpha_{p}=\frac{0.0079(\tilde{V}-71.5)}{1-\exp \left(\frac{71.5-\tilde{V}}{23.6}\right)} ; \beta_{p}=\frac{-0.00478(\tilde{V}-3.9)}{1-\exp \left(\frac{\tilde{V}-3.9}{21.8}\right)}$

The other constants are:

$P_{n a}=0.00704 \mathrm{~cm} / \mathrm{s}$

$[N a]_{0}=154 \mathrm{mmol} / 1 ;[N a]_{i}=30 \mathrm{mmol} / \mathrm{l}$ 


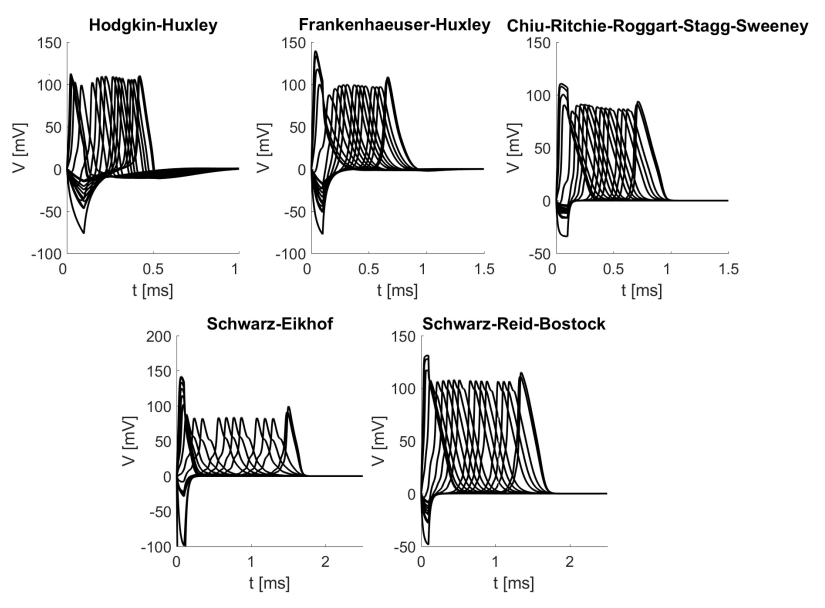

Fig. 12 Example of propagating action potentials generated by cathode-make stimulation in the studied membrane models. Membrane voltage plots at subsequent nodes of Ranvier are shown. The action potential slows down at the hyperpolarized region close the the virtual anodes, and speeds up at the neural terminations.

Acknowledgements This work was carried out using the Supercomputer Infrastructure (STEVIN) at Ghent University,

funded by Ghent University, the Flemish Supercomputer Center (VSC), the Hercules Foundation and the Flemish Government department EWI.

This research was funded by the FWO-project G046816N. T. Tarnaud is a PhD Fellow of the FWO-V (SB) (Research Foundation Flanders, Belgium). E. Tanghe is a Post-Doctoral Fellow of the FWO-V (Research Foundation Flanders, Belgium).

\section{References}

1. Blair, H.A.: On the intensity-time relations for stimulation by electric currents. i. The Journal of general physiology 15(6), 709-729 (1932)

2. Blight, A.: Computer simulation of action potentials and afterpotentials in mammalian myelinated axons: the case for a lower resistance myelin sheath. Neuroscience 15(1), 13-31 (1985)

3. Brette, R.: What is the most realistic single-compartment model of spike initiation? PLoS Comput Biol 11(4), e1004,114 (2015)

4. Brunel, N., van Rossum, M.C.: Quantitative investigations of electrical nerve excitation treated as polarization. Biological Cybernetics 97(5), 341-349 (2007)

5. Cartee, L.A.: Evaluation of a model of the cochlear neural membrane. ii: Comparison of model and physiological measures of membrane properties measured in response to intrameatal electrical stimulation. Hearing research 146(1), 153-166 (2000)

6. Cartee, L.A.: Spiral ganglion cell site of excitation ii: numerical model analysis. Hearing research 215(1), 22-30 (2006)
7. Chiu, S., Ritchie, J., Rogart, R., Stagg, D.: A quantitative description of membrane currents in rabbit myelinated nerve. The Journal of physiology 292, 149 (1979)

8. Crank, J., Nicolson, P.: A practical method for numerical evaluation of solutions of partial differential equations of the heat-conduction type. In: Mathematical Proceedings of the Cambridge Philosophical Society, vol. 43, pp. 5067. Cambridge Univ Press (1947)

9. Fitzhugh, R.: Computation of impulse initiation and saltatory conduction in a myelinated nerve fiber. Biophysical journal 2(1), 11-21 (1962)

10. Frankenhaeuser, B., Huxley, A.: The action potential in the myelinated nerve fibre of xenopus laevis as computed on the basis of voltage clamp data. The Journal of Physiology 171(2), 302 (1964)

11. Fribance, S., Wang, J., Roppolo, J.R., de Groat, W.C., Tai, C.: Axonal model for temperature stimulation. Journal of Computational Neuroscience 41(2), 185-192 (2016)

12. Gerstner, W., Naud, R.: How good are neuron models? Science 326(5951), 379-380 (2009)

13. Hodgkin, A.L., Huxley, A.F.: Propagation of electrical signals along giant nerve fibres. Proceedings of the Royal Society of London. Series B, Biological Sciences pp. 177183 (1952)

14. ICNIRP: Guidelines for limiting exposure to time-varying electric and magnetic fields ( $1 \mathrm{hz}$ to $100 \mathrm{khz})$. Health Physics 99(6), 818-836 (2010)

15. IEEE Standards Coordinating Committee 28: IEEE Standard for Safety Levels with Respect to Human Exposure to Radio Frequency Electromagnetic Fields, $3 \mathrm{kHz}$ to $300 \mathrm{GHz}$. Institute of Electrical and Electonics Engineers, Incorporated (1992)

16. Izhikevich, E.M.: Which model to use for cortical spiking neurons? IEEE transactions on neural networks 15(5), 1063-1070 (2004)

17. Izhikevich, E.M., Edelman, G.M.: Large-scale model of mammalian thalamocortical systems. Proceedings of the national academy of sciences 105(9), 3593-3598 (2008)

18. Lapicque, L.: Recherches quantitatives sur lexcitation électrique des nerfs traitée comme une polarisation. J. Physiol. Pathol. Gen 9(1), 620-635 (1907)

19. Markram, H., Muller, E., Ramaswamy, S., Reimann, M.W., Abdellah, M., Sanchez, C.A., Ailamaki, A., Alonso-Nanclares, L., Antille, N., Arsever, S., et al.: Reconstruction and simulation of neocortical microcircuitry. Cell 163(2), 456-492 (2015)

20. McIntyre, C.C., Grill, W.M.: Sensitivity analysis of a model of mammalian neural membrane. Biological cybernetics 79(1), 29-37 (1998)

21. McIntyre, C.C., Richardson, A.G., Grill, W.M.: Modeling the excitability of mammalian nerve fibers: influence of afterpotentials on the recovery cycle. Journal of neurophysiology 87(2), 995-1006 (2002)

22. McNeal, D.R.: Analysis of a model for excitation of myelinated nerve. IEEE Transactions on Biomedical Engineering 23(4), 329-337 (1976)

23. O'Brien, G.E., Rubinstein, J.T.: The development of biophysical models of the electrically stimulated auditory nerve: Single-node and cable models. Network: Computation in Neural Systems 27(2-3), 135-156 (2016)

24. Ranjan, R., Khazen, G., Gambazzi, L., Ramaswamy, S., Hill, S.L., Schürmann, F., Markram, H.: Channelpedia: an integrative and interactive database for ion channels. Frontiers in neuroinformatics 5, 1-8 (2011) 
25. Rattay, F.: Analysis of models for external stimulation of axons. IEEE transactions on biomedical engineering (10), 974-977 (1986)

26. Rattay, F.: Current distance relations for fiber stimulation with pointsources. IEEE Transactions on Biomedical Engineering 55(3), 1122-1127 (2008)

27. Rattay, F., Aberham, M.: Modeling axon membranes for functional electrical stimulation. IEEE Transactions on Biomedical Engineering 40(12), 1201-1209 (1993)

28. Reilly, J.P.: Survey of numerical electrostimulation models. Physics in medicine and biology 61(12), 4346 (2016)

29. Reilly, J.P., Diamant, A.M.: Electrostimulation: theory, applications, and computational model. Artech House (2011)

30. Reilly, J.P., Freeman, V.T., Larkin, W.D.: Sensory effects of transient electrical stimulation-evaluation with a neuroelectric model. IEEE Transactions on Biomedical Engineering 32(12), 1001-1011 (1985)

31. Reilly, J.P., Hirata, A.: Low-frequency electrical dosimetry: research agenda of the ieee international committee on electromagnetic safety. Physics in medicine and biology 61(12), 138-149 (2016)

32. Richardson, A., McIntyre, C., Grill, W.: Modelling the effects of electric fields on nerve fibres: influence of the myelin sheath. Medical and Biological Engineering and Computing 38(4), 438-446 (2000)

33. Rubinstein, J.T.: Analytical theory for extracellular electrical stimulation of nerve with focal electrodes. ii. passive myelinated axon. Biophysical journal 60(3), 538-555 (1991)

34. Šarolić, A., Živković, Z., Reilly, J.: Measurement and simulation of unmyelinated nerve electrostimulation: Lumbricus terrestris experiment and numerical model. Physics in medicine and biology 61(12), 4364-4375 (2016)

35. Schwarz, J.R., Eikhof, G.: Na currents and action potentials in rat myelinated nerve fibres at 20 and $37 \mathrm{c}$. Pflügers Archiv European Journal of Physiology 409(6), 569-577 (1987)

36. Schwarz, J.R., Reid, G., Bostock, H.: Action potentials and membrane currents in the human node of ranvier. Pflügers Archiv European Journal of Physiology 430(2), 283-292 (1995)

37. Stephanova, D., Bostock, H.: A distributed-parameter model of the myelinated human motor nerve fibre: temporal and spatial distributions of action potentials and ionic currents. Biological cybernetics 73(3), 275-280 (1995)

38. Sweeney, J., Mortimer, J., Durand, D.: Modeling of mammalian myelinated nerve for functional neuromuscular stimulation. In: IEEE 9th Annual Conference of the Engineering in Medicine and Biology Society, vol. 3, pp. 1577-1578 (1987)

39. Tai, C., De Groat, W.C., Roppolo, J.R.: Simulation analysis of conduction block in unmyelinated axons induced by high-frequency biphasic electrical currents. IEEE Transactions on Biomedical Engineering 52(7), 13231332 (2005)

40. Tasaki, I.: New measurements of the capacity and the resistance of the myelin sheath and the nodal membrane of the isolated frog nerve fiber. Am J Physiol 181(3), 639-650 (1955)

41. Weiss, G.: Sur la possibilite de rendre comparables entre eux les appareils servant a l'excitation electrique. Archives Italiennes de Biologie 35(1), 413-445 (1901)

42. Zhang, X., Roppolo, J.R., De Groat, W.C., Tai, C.: Mechanism of nerve conduction block induced by high- frequency biphasic electrical currents. IEEE Transactions on Biomedical Engineering 53(12), 2445-2454 (2006)

43. Zhao, S., Yang, G., Wang, J., Roppolo, J.R., de Groat, W.C., Tai, C.: Effect of non-symmetric waveform on conduction block induced by high-frequency (khz) biphasic stimulation in unmyelinated axon. Journal of computational neuroscience 37(2), 377-386 (2014) 


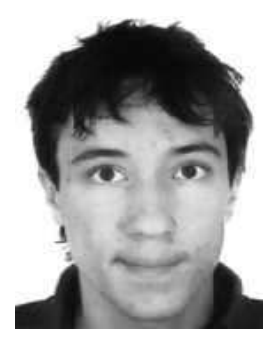

Thomas Tarnaud is a Ph.D. student in wireless, acoustics, environment and expert systems (UGentimec). His current research involves computational modeling of neurostimulation.

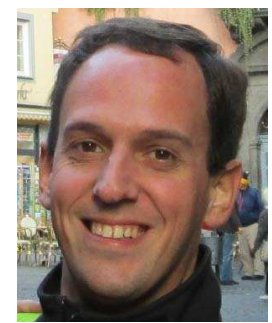

Wout Joseph is a professor in the domain of experimental characterization of wireless communication systems: UGent. He specializes in EM exposure, propagation, and wireless performance analysis.

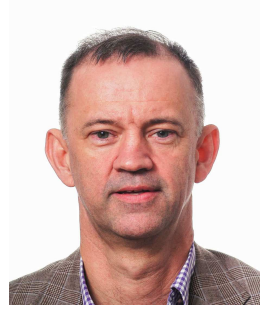

Luc Martens is a professor at UGent. His interests are in modeling and measurement of electromagnetic channels and exposure and energy consumption

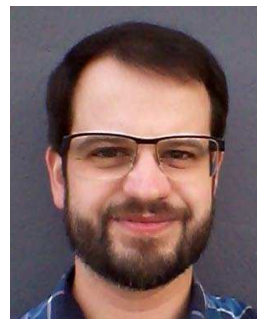

Emmeric Tanghe is a postdoc toral fellow of the FWO-V in radio propagation and parttime professor in medical applications of electromagnetic fields in and around the human body. 